\title{
General Franklin systems as bases in $H^{1}[0,1]$
}

\author{
by \\ Gegham G. Gevorkyan (Yerevan) and Anna Kamont (Sopot)
}

\begin{abstract}
By a general Franklin system corresponding to a dense sequence of knots $\mathcal{T}=\left(t_{n}, n \geq 0\right)$ in $[0,1]$ we mean a sequence of orthonormal piecewise linear functions with knots $\mathcal{T}$, that is, the $n$th function of the system has knots $t_{0}, \ldots, t_{n}$. The main result of this paper is a characterization of sequences $\mathcal{T}$ for which the corresponding general Franklin system is a basis or an unconditional basis in $H^{1}[0,1]$.
\end{abstract}

\section{INTRODUCTION}

The classical Franklin system is a complete orthonormal system consisting of piecewise linear continuous functions with dyadic knots. It was introduced by Ph. Franklin [7] in 1928 as an example of a complete orthonormal system which is a basis in $C[0,1]$. Since then, it has been studied by many authors from different points of view, and various extensions and generalizations of this system have been considered. In particular, it is well known that this system is an unconditional basis in $L^{p}[0,1], 1<p<\infty$ (see S. V. Bochkarev [1]) and in $H^{1}[0,1]$ (see P. Wojtaszczyk [17]).

The present paper is a continuation of [10]. In both papers, we study the properties of a generalization of the classical Franklin system obtained by replacing the dyadic knots by a general sequence of knots. Given a sequence of knots $\mathcal{T}=\left(t_{n}, n \geq 0\right)$ in $[0,1]$ admitting at most double knots and dense in $[0,1]$, by a general Franklin system corresponding to $\mathcal{T}$ we mean the complete orthonormal system consisting of piecewise linear functions with

2000 Mathematics Subject Classification: 42C10, 46E30.

Key words and phrases: general Franklin system, basis, unconditional basis, $H^{1}$ space.

Part of this work was done when A. Kamont was visiting the Institute of Mathematics of the National Academy os Sciences of Armenia in May 2003. The paper was prepared for publication when A. Kamont was visiting Institut für Analysis der Johannes Kepler Universität in Linz in the Autumn of 2003. A. Kamont's stay and travel to Linz were supported by FWF project P150907-N08 and P. Wojtaszczyk's professor subsidy of the Foundation of Polish Science. 
knots $\mathcal{T}$ (see Section 2.1 for the precise definition). We are interested in the properties of this system as a basis in various function spaces. For the spaces $L^{p}[0,1], 1<p<\infty$, or $C[0,1]$, these properties are now fully understood: Z. Ciesielski [4] has proved that the $L^{\infty}$-norm of the orthogonal projection onto the space of piecewise linear functions with arbitrary knots does not exceed 3, which implies that each general Franklin system is a basis in $L^{p}[0,1], 1 \leq p<\infty$, and if all knots are simple (so all functions of the system are continuous), it is a basis in $C[0,1]$. The question of unconditionality of this basis in $L^{p}[0,1], 1<p<\infty$, has also been considered. In G. G. Gevorkyan and A. Kamont [9] and G. G. Gevorkyan and A. A. Sahakian [11], some partial answers have been obtained, under additional assumptions on the structure (in both papers, quasi-dyadic structure) and regularity of the sequence of knots. Finally, in [10], we have proved that for each sequence of knots the corresponding general Franklin system is an unconditional basis in $L^{p}[0,1], 1<p<\infty$.

In the present paper, we are interested in the properties of the general Franklin system as a basis in the real Hardy space $H^{1}[0,1]$. We have already studied this question in [9] and proved that, for a quasi-dyadic sequence of knots, the general Franklin system is a basis in $H^{1}[0,1]$ if and only if the sequence of knots satisfies a strong regularity condition; moreover, if it is a basis in $H^{1}[0,1]$, then it is an unconditional basis. However, the result of [10] does not require any assumption on the structure of the sequence of knots. This has been the motivation for returning to the $H^{1}[0,1]$ case and seeing what happens if we do not assume the quasi-dyadic structure. In this generality, we have arrived at two conditions on regularity of knots, which we call strong regularity for pairs and strong regularity (the latter being the same as in [9]). We prove that strong regularity for pairs is a necessary and sufficient condition for the general Franklin system to be a basis in $H^{1}[0,1]$, and that strong regularity is a necessary and sufficient condition for the system to be an unconditional basis in $H^{1}[0,1]$ (for definitons etc. see Section 2.2). These are the main results of this paper, Theorems 2.1 and 2.2. Let us remark that for quasi-dyadic sequences of partitions strong regularity and strong regularity for pairs coincide.

Finally, as the paper concerns unconditional bases in $H^{1}$, let us briefly recall the main results in this direction. The existence of an unconditional basis in $H^{1}$ was first proved by B. Maurey [13], but his proof was not constructive. The first explicit construction is due to L. Carleson [2]. Then P. Wojtaszczyk proved that the classical Franklin system is an unconditional basis in $H^{1}$, and Sung-Yung A. Chang and Z. Ciesielski [3] proved this for spline systems of higher orders (with dyadic knots). Analogous results are also well known for (sufficiently regular) wavelets (cf. e.g. books [14] or [18]). 
The paper is organized as follows. In Section 2 we give the basic definitions and formulate the main results, Theorems 2.1 and 2.2. In Section 3 we collect some properties of a single general Franklin function and of a general Franklin system. Section 4 concerns the relations between unconditional convergence in $L^{1}$ of a general Franklin series, integrability of square and maximal functions of such series and being the Fourier-Franklin series of a function from $H^{1}$ (see conditions (A)-(D) there). The results in this direction are: Fact 4.2 and Propositions 4.3, 4.5, 4.6 and 4.7; see also Corollary 5.3. Section 5 contains the proofs of the main results. In addition, we note that if a general Franklin system (normalized in $H^{1}$ ) is an unconditional basis in $H^{1}[0,1]$, then it is also a greedy basis in this space (see Corollary 5.4).

Notation. Throughout the paper, the following notation is used. For a set $A \subset[0,1]$, we denote by $\chi_{A}$ the characteristic function of $A$, by $|A|$ its Lebesgue measure, and by $A^{\mathrm{c}}$ its complement in $[0,1]$; for $t \in[0,1], \operatorname{dist}(t, A)$ is the distance from $t$ to $A$. For a finite set $B, \# B$ denotes the number of elements of $B$. For a function $f:[0,1] \rightarrow \mathbb{R}, \mathcal{M} f$ is the Hardy-Littlewood maximal function of $f$. We use the notation $x \vee y=\max (x, y), x \wedge y=$ $\min (x, y)$. Finally, $a \sim b$ means that there are positive constants $c_{1}, c_{2}$, independent of the variables of $a, b$, such that $c_{1} a \leq b \leq c_{2} a$, and $a \sim_{\gamma} b$ means that the implied constants may depend only on the parameter $\gamma$, but not on other variables of $a, b$.

\section{BASIC DEFINITIONS AND FORMULATION OF THE MAIN RESULTS}

2.1. Basic definitions. Let us recall the definition of real Hardy spaces $H^{1}[0,1]$. We use the atomic definition, first introduced in [6].

A function $a:[0,1] \rightarrow \mathbb{R}$ is called an atom if either $a=1$, or there is an interval $\Gamma \subset[0,1]$ such that $\operatorname{supp} a \subset \Gamma, \sup |a| \leq 1 /|\Gamma|$ and $\int_{0}^{1} a(u) d u=0$. A function $f \in L^{1}[0,1]$ is said to belong to $H^{1}[0,1]$ if there are atoms $a_{j}$ and real coefficients $c_{j}, j \in \mathbb{N}$, with $\sum_{j=1}^{\infty}\left|c_{j}\right|<\infty$ such that $f=\sum_{j=1}^{\infty} c_{j} a_{j}$. The norm in $H^{1}[0,1]$ is defined as $\|f\|_{H^{1}}=\inf \left(\sum_{j=1}^{\infty}\left|c_{j}\right|\right)$, where the infimum is taken with respect to all atomic decompositions of $f$.

Next, let us recall the definitions of a general Franklin function and a general Franklin system.

Let $\sigma=\left(s_{i}, 0 \leq i \leq N\right)$ be a partition of $[0,1]$, admitting at most double knots, i.e., a sequence of knots in $[0,1]$ such that

$$
\left\{\begin{array}{l}
0=s_{0}<s_{1} \leq \cdots \leq s_{N-1}<s_{N}=1 \\
s_{i}<s_{i+2} \text { for } 0 \leq i \leq N-2
\end{array}\right.
$$


Denote by $S(\sigma)$ the space of piecewise linear functions on $[0,1]$ with knots $\sigma$, i.e., linear on each $\left(s_{i}, s_{i+1}\right)$, left-continuous at each $s_{i}$ (and right-continuous at $\left.s_{0}=0\right)$ and continuous at each $s_{i}, 1 \leq i \leq N-1$, satisfying $s_{i-1}<s_{i}<$ $s_{i+1}$. By $\left\{N_{\sigma, i}, 0 \leq i \leq N\right\}$ we denote the usual $B$-spline basis corresponding to the knots $\sigma$ : if $s_{i}$ is a simple knot in $\sigma$ then $N_{\sigma, i}$ is the unique piecewise linear and continuous function with knots $\sigma$ satisfying $N_{\sigma, i}\left(s_{k}\right)=\delta_{i, k}$; when $s_{i-1}=s_{i}$, then $N_{\sigma, i-1}, N_{\sigma, i}$ are the unique piecewise linear functions with knots $\sigma$, continuous and taking value 0 at all knots different from the double knot $s_{i-1}=s_{i}$, left-continuous at $s_{i-1}=s_{i}$, and satisfying $N_{\sigma, i-1}\left(s_{i-1}\right)=1$, $\lim _{s \rightarrow s_{i-1}^{+}} N_{\sigma, i-1}(s)=0, N_{\sigma, i}\left(s_{i}\right)=0$ and $\lim _{s \rightarrow s_{i}^{+}} N_{\sigma, i}(s)=1$.

Now, let $\sigma=\left(s_{i}, 0 \leq i \leq N\right)$ and $\sigma^{*}=\left(s_{i}^{*}, 0 \leq i \leq N+1\right)$ be a pair of partitions of $[0,1]$ satisfying $(2.1)$ and such that $\sigma^{*}$ is obtained from $\sigma$ by adding one knot $s^{*}$. Note that $s^{*}$ may be different from all knots of $\sigma$ (in this case, for some $i$, we have $s^{*}=s_{i}^{*}$ and $s_{i-1}^{*}<s_{i}^{*}<s_{i+1}^{*}$ ), or for some $i, s^{*}=s_{i}\left(\right.$ then $\left.s_{i-1}^{*}<s_{i}^{*}=s^{*}=s_{i+1}^{*}<s_{i+2}^{*}\right)$. In each case, there is a unique function $\varphi \in S\left(\sigma^{*}\right)$ such that $\varphi$ is orthogonal to $S(\sigma)$ in $L^{2}[0,1]$, $\|\varphi\|_{2}=1$ and $\varphi\left(s^{*}\right)>0$. This function $\varphi$ is called the general Franklin function corresponding to the pair of partitions $\left(\sigma, \sigma^{*}\right)$.

Now, we turn to sequences of partitions and general Franklin systems.

Let $\mathcal{T}=\left(t_{i}, i \geq 0\right)$ be a sequence of knots in $[0,1]$, admitting at most double knots, with $t_{0}=0, t_{1}=1, t_{i} \in(0,1)$ for $i \geq 2$ and dense in $[0,1]$. Such a sequence of knots is called admissible. For $n \geq 1$, let $\mathcal{T}_{n}=\left(t_{i}, 0 \leq i \leq n\right)$, and let $\pi_{n}=\left(0=t_{n, 0}<t_{n, 1} \leq \cdots \leq t_{n, n-1}<t_{n, n}=1\right)$ be a partition of $[0,1]$ obtained by nondecreasing rearrangement of $\mathcal{T}_{n}$. Let us introduce some notation, which will be used throughout the paper:

$$
I_{n, i}=\left[t_{n, i-1}, t_{n, i}\right], \quad \lambda_{n, i}=\left|I_{n, i}\right|=t_{n, i}-t_{n, i-1} .
$$

Note that each $\pi_{n}$ satisfies (2.1), and $\pi_{n}$ is obtained from $\pi_{n-1}$ by adding one knot $t_{n}$.

Definition 2.1. Let $\mathcal{T}$ be an admissible sequence of knots. A general Franklin system with knots $\mathcal{T}$ is a sequence of functions $\left\{f_{n}, n \geq 0\right\}$ given by

$$
f_{0}(t)=1, \quad f_{1}(t)=\sqrt{3}(2 t-1),
$$

and for $n \geq 2, f_{n}$ is the general Franklin function corresponding to $\left(\pi_{n-1}, \pi_{n}\right)$.

2.2. The main results. For the main results, we need to formulate two regularity conditions for $\mathcal{T}$, and this is done with the notation of (2.2).

Definition 2.2. Let $\mathcal{T}$ be an admissible sequence of knots. We say that $\mathcal{T}$ satisfies the strong regularity condition with parameter $\gamma \geq 1$ if for each $n \geq 1$ and $2 \leq i \leq n$,

$$
\frac{1}{\gamma} \lambda_{n, i-1} \leq \lambda_{n, i} \leq \gamma \lambda_{n, i-1}
$$


Definition 2.3. Let $\mathcal{T}$ be an admissible sequence of knots. We say that $\mathcal{T}$ satisfies the strong regularity condition for pairs with parameter $\gamma \geq 1$ if for each $n \geq 2$ and $1 \leq i \leq n$,

$$
\frac{1}{\gamma}\left(\lambda_{n, i-1}+\lambda_{n, i}\right) \leq \lambda_{n, i}+\lambda_{n, i+1} \leq \gamma\left(\lambda_{n, i-1}+\lambda_{n, i}\right),
$$

with the convention $\lambda_{n, 0}=\lambda_{n, n+1}=0$.

Now, we formulate the main result of this paper, Theorems 2.1 and 2.2, which characterize those sequences of partitions for which the corresponding Franklin system is a basis or an unconditional basis in $H^{1}[0,1]$.

THEOREM 2.1. Let $\mathcal{T}$ be an admissible sequence of knots in $[0,1]$ with the corresponding Franklin system $\left\{f_{n}, n \geq 0\right\}$. Then $\left\{f_{n}, n \geq 0\right\}$ is a basis in $H^{1}[0,1]$ if and only if $\mathcal{T}$ satisfies the strong regularity condition for pairs with some parameter $\gamma>1$.

THEOREM 2.2. Let $\mathcal{T}$ be an admissible sequence of knots in $[0,1]$ with the corresponding Franklin system $\left\{f_{n}, n \geq 0\right\}$. Then $\left\{f_{n}, n \geq 0\right\}$ is an unconditional basis in $H^{1}[0,1]$ if and only if $\mathcal{T}$ satisfies the strong regularity condition with some parameter $\gamma>1$.

2.2.1. Comments. In [9] we have discussed general Franklin systems as bases in $H^{1}[0,1]$, but only for quasi-dyadic sequences of knots. By this we mean the following: consider a sequence of partitions $\mathcal{P}_{j}=\left\{\tau_{j, k}, 0 \leq k \leq 2^{j}\right\}$, $j \geq 0$, such that $0=\tau_{j, 0}<\tau_{j, 1}<\cdots<\tau_{j, 2^{j}}=1$ and $\tau_{j+1,2 k}=\tau_{j, k}$ for all $j, k, 0 \leq k \leq 2^{j}$, i.e. between each pair of knots of $\mathcal{P}_{j}$, one new knot of $\mathcal{P}_{j+1}$ is inserted. Putting $t_{0}=0, t_{1}=1$ and $t_{n}=\tau_{j, 2 k-1}$ for $n=2^{j}+k$ with $j \geq 0$ and $1 \leq k \leq 2^{j}$, we get an admissible sequence $\mathcal{T}=\left(t_{n}, n \geq 0\right)$ of simple knots with quasi-dyadic structure.

The first of the above regularity conditions, the strong regularity condition, has been used in [9]. Theorem 5.3 of [9] states that for quasi-dyadic sequences of knots, a general Franklin system is a basis in $H^{1}[0,1]$ iff it is an unconditional basis in $H^{1}[0,1]$, and both these conditions are equivalent to the strong regularity of the sequence of knots. It turns out that for general Franklin systems without any structural constraints on the corresponding sequence of knots, the properties of being a basis in $H^{1}[0,1]$ and being an unconditional basis in $H^{1}[0,1]$ are no longer equivalent.

Clearly, for quasi-dyadic sequences of knots strong regularity and strong regularity for pairs are equivalent. In addition, the quasi-dyadic structure of a strongly regular sequence of knots implies the following polynomial propagation of lengths of intervals: there are $\alpha_{\gamma}$ and $C_{\gamma}$, depending only on the regularity parameter $\gamma$, such that for all $j \geq 0$ and $1 \leq k, l \leq 2^{j}$,

$$
C_{\gamma}^{-1}(|k-l|+1)^{-\alpha_{\gamma}} \lambda_{2^{j}, k} \leq \lambda_{2^{j}, l} \leq C_{\gamma}(|k-l|+1)^{\alpha_{\gamma}} \lambda_{2^{j}, k}
$$


(cf. Proposition 2.6(ii) of [9]). This property has been used in [9], and it enabled us to treat also the case of $H^{p}[0,1]$ with $p<1$. However, for general strongly regular sequences of knots, an analogue of (2.3) need not hold. The proofs in this paper do not make use of (2.3), but we have to restrict ourselves to the case $p=1$. As in [10], the technique of proofs depends on the analysis of the canonical intervals associated with Franklin functions (cf. Section 3.1).

Finally, note that strong regularity implies that all knots of $\mathcal{T}$ are simple, while double knots are allowed for sequences enjoying strong regularity for pairs. Both strong regularity and strong regularity for pairs can be encountered in the context of spline approximation: strong regularity is just the boundedness of the local mesh ratio, while strong regularity for pairs is equivalent to the boundedness of the ratios of the lengths of the supports of the basic functions $N_{\pi_{n}, i}, 0 \leq i \leq n$.

\section{BASIC PROPERTIES OF A GENERAL FRANKLIN SYSTEM}

3.1. Properties of a single Franklin function. We recall some definitions and estimates for a general Franklin function. For a more detailed description and proofs we refer to Section 3.1 of [10].

As in [10], to simplify notation, assume that

$$
\pi=\left\{0=\tau_{-k}<\tau_{-k+1} \leq \cdots \leq \tau_{-1}<\tau_{1} \leq \cdots \leq \tau_{l-1}<\tau_{l}=1\right\},
$$

and $\pi^{*}=\pi \cup\{\tau\}$ with $\tau_{-1}<\tau=\tau_{0} \leq \tau_{1}$ (with $\tau_{i}<\tau_{i+2}$ ). As in Section 2.1, $\varphi$ denotes the general Franklin function corresponding to $\left(\pi, \pi^{*}\right)$. In this section, we use the notation

$$
\lambda_{i}=\tau_{i}-\tau_{i-1} .
$$

First, we recall the definition of a "canonical" interval $J$ associated with $\varphi$ (cf. Section 3.1 of [10]). The definition depends on whether $\tau$ is a simple or a double knot of $\pi^{*}$.

First, let $\tau$ be a simple knot of $\pi^{*}$, i.e. $\tau_{-1}<\tau=\tau_{0}<\tau_{1}$. Consider the intervals

$$
I=\left[\tau_{-1}, \tau_{1}\right], \quad I^{-}=\left[\tau_{-2}, \tau_{0}\right], \quad I^{+}=\left[\tau_{0}, \tau_{2}\right],
$$

and set

$$
\nu=|I|, \quad \nu^{-}=\left|I^{-}\right|, \quad \nu^{+}=\left|I^{+}\right|, \quad \mu=\min \left(\nu^{-}, \nu, \nu^{+}\right) .
$$

(In case $k=1$ or $l=1$, we take $\tau_{-2}=0$ or $\tau_{2}=1$, respectively.) Now, choose $I^{*}=\left[\tau_{i^{*}}, \tau_{i^{*}+2}\right]$ to be one of the intervals $I^{-}, I, I^{+}$such that $\mu=\left|I^{*}\right|$, and consider its left and right parts $I^{*, l}=\left[\tau_{i^{*}}, \tau_{i^{*}+1}\right], I^{*, r}=\left[\tau_{i^{*}+1}, \tau_{i^{*}+2}\right]$. Finally, let $J$ be one of the intervals $I^{*, l}, I^{*, r}$ such that $|J|=\max \left(\left|I^{*, l}\right|,\left|I^{*, r}\right|\right)$. Note that in this case $|J| \leq \mu \leq 2|J|$. In what follows, we also need the following 
notation:

$$
\tau^{-}=\tau_{-1}, \quad \tau^{+}=\tau_{1}
$$

Now, let $\tau$ be a double knot of $\pi^{*}$, i.e. $\tau_{-1}<\tau=\tau_{0}=\tau_{1}<\tau_{2}$. Consider the intervals $I^{-}=\left[\tau_{-1}, \tau_{0}\right], I^{+}=\left[\tau_{1}, \tau_{2}\right]$ and let $\mu=\min \left(\left|I^{-}\right|,\left|I^{+}\right|\right)$. Now, take as $J$ one of $I^{-}, I^{+}$such that $|J|=\mu$. Moreover, we put $\tau^{-}=\tau_{-1}$ and $\tau^{+}=\tau_{2}$.

Pointwise estimates for a general Franklin function have been discussed in [9], [11] and [10]. We will need some estimates from [10, Propositions 3.1 and 3.2]. The estimates are formulated in terms of the coefficients $\xi_{i}$ of the representation $\varphi=\sum_{i=-k}^{l} \xi_{i} N_{\pi^{*}, i}$. Note that if $\tau_{i}$ is a simple knot of $\pi^{*}$ then $\xi_{i}=\varphi\left(\tau_{i}\right)$, and if $\tau_{i-1}=\tau_{i}$, then $\xi_{i-1}=\varphi\left(\tau_{i-1}\right)=\lim _{t \rightarrow \tau_{i-1}^{-}} \varphi(t)$ and $\xi_{i}=\lim _{t \rightarrow \tau_{i}^{+}} \varphi(t)$. As $\varphi$ is linear on each $\left(\tau_{i-1}, \tau_{i}\right)$, it is clear that estimates for $\xi_{i-1}, \xi_{i}$ imply estimates for $\varphi$ and $\varphi^{\prime}$ on $\left(\tau_{i-1}, \tau_{i}\right)$.

To formulate the estimates, we need some additional notation. As in [10], for $x, y \in[0,1]$, we denote by $d_{\pi^{*}}(x, y)$ the number of knots of $\pi^{*}$ between $x$ and $y$, counting multiplicities, i.e.

$$
d_{\pi^{*}}(x, y)=\#\left\{i: x \wedge y \leq \tau_{i} \leq x \vee y\right\}
$$

By $d_{\pi^{*}}(x)$ we denote the number of knots of $\pi^{*}$ between $x$ and $J$, counting multiplicities and endpoints of $J$, with the understanding that $d_{\pi^{*}}(x)=0$ when $x \in J$. Similarly, for an interval $V \subset[0,1]$, we denote by $d_{\pi^{*}}(V)$ the number of knots of $\pi^{*}$ between $V$ and $J$, counting multiplicities and endpoints of $J$ or $V$, with the understanding that $d_{\pi^{*}}(V)=0$ whenever $V \cap J \neq \emptyset$.

Proposition 3.1. Let $\pi^{*}=\pi \cup\left\{\tau_{0}\right\}$ be as described above, and let $\varphi$ be the general Franklin function corresponding to $\left(\pi, \pi^{*}\right), \varphi=\sum_{i=-k}^{l} \xi_{i} N_{\pi^{*}, i}$. If $\tau=\tau_{0}$ is a simple knot of $\pi^{*}$, then

$$
\begin{cases}\|\varphi\|_{p} \sim \mu^{1 / p-1 / 2}, & 1 \leq p \leq \infty \\ \left|\xi_{-1}\right| \sim \mu^{1 / 2} / \nu^{-}, & \left|\xi_{0}\right| \sim \mu^{1 / 2} / \nu, \quad\left|\xi_{1}\right| \sim \mu^{1 / 2} / \nu^{+}\end{cases}
$$

with the implied constants independent of $\left(\pi, \pi^{*}\right)$ and $p$.

If $\tau=\tau_{0}=\tau_{1}$ is a double knot of $\pi^{*}$, then

$$
\|\varphi\|_{p} \sim \mu^{1 / p-1 / 2}, \quad 1 \leq p \leq \infty, \quad\left|\xi_{0}\right| \sim \mu^{1 / 2} / \lambda_{0}, \quad\left|\xi_{1}\right| \sim \mu^{1 / 2} / \lambda_{2},
$$

with the implied constants independent of $\left(\pi, \pi^{*}\right)$ and $p$.

In both cases (i.e. $\tau$ being either a simple or a double knot of $\pi^{*}$ )

$$
\|\varphi\|_{L^{p}(J)} \sim\|\varphi\|_{p} \sim|J|^{1 / p-1 / 2}, \quad 1 \leq p \leq \infty,
$$

with the implied constants independent of $p, \pi, \pi^{*}$. In addition, we have $\left|\xi_{i}\right|=$ $(-1)^{|i|} \xi_{i}$ and the following localization of the support of $\varphi$ : if $\tau_{i-1}=\tau_{i} \leq \tau^{-}$ (respectively, $\left.\tau^{+} \leq \tau_{i}=\tau_{i+1}\right)$, then $\operatorname{supp} \varphi \subset\left[\tau_{i}, 1\right]$ (respectively, $\operatorname{supp} \varphi \subset$ $\left.\left[0, \tau_{i}\right]\right)$. 
Moreover, there is a constant $C$, independent of $\pi, \pi^{*}$, such that

$$
\left|\xi_{i}\right| \leq C\left(\frac{2}{3}\right)^{d_{\pi^{*}\left(\tau_{i}\right)}} \frac{|J|^{1 / 2}}{|J|+\operatorname{dist}\left(\tau_{i}, J\right)+\tau_{i+1}-\tau_{i-1}} \quad \text { for all } i .
$$

In addition, with $\epsilon=(\sqrt{2}+1) / 3$, in the case when $\tau$ is a simple knot in $\pi^{*}$, we have the following estimates:

(a) for $i \leq i+s \leq-1$ :

$$
\begin{aligned}
& \int_{0}^{\tau_{i}}|\varphi(t)| d t \leq \frac{\epsilon^{s}}{1-\epsilon} \int_{\tau_{i+s-1}}^{\tau_{i+s}}|\varphi(t)| d t \\
& \int_{0}^{\tau_{i}}|\varphi(t)| d t \leq \frac{\epsilon^{|i|}}{1-\epsilon}\|\varphi\|_{1}
\end{aligned}
$$

(b) for $1 \leq i-s \leq i$ :

$$
\begin{aligned}
& \int_{\tau_{i}}^{1}|\varphi(t)| d t \leq \frac{\epsilon^{s}}{1-\epsilon} \int_{\tau_{i-s}}^{\tau_{i-s+1}}|\varphi(t)| d t \\
& \int_{\tau_{i}}^{1}|\varphi(t)| d t \leq \frac{\epsilon^{|i|}}{1-\epsilon}\|\varphi\|_{1},
\end{aligned}
$$

If $\tau$ is a double knot of $\pi^{*}$, then (a) holds for $i \leq i+s \leq 0$, and (b) holds for $i \geq i-s \geq 1$.

3.2. Properties of a general Franklin system. Let $\mathcal{T}=\left\{t_{n}, n \geq 0\right\}$ be an admissible sequence of knots with the corresponding Franklin system $\left\{f_{n}, n \geq 0\right\}$. By $I_{n}, I_{n}^{*}, J_{n}, \mu_{n}, d_{n}$ etc. we denote the intervals and quantities defined above for a general Franklin function and corresponding to the function $f_{n}$ and partition $\pi_{n}$. In addition, $t_{n}^{-}, t_{n}^{+}$correspond to $t_{n}$ and $\pi_{n-1}$ in the same way as $\tau^{-}, \tau^{+}$correspond to $\tau$ and $\pi$ in Section 3.1.

The following properties of a general Franklin system have been proved in [10, Lemmas 3.4 and 3.5]:

LEMMA 3.2. Let $\mathcal{T}=\left(t_{n}, n \geq 0\right)$ be an admissible sequence of knots with the corresponding Franklin system $\left\{f_{n}, n \geq 0\right\}$. Let $k, l \geq 0$ be such that $t_{k} \leq t_{l}$ and there is no $i \leq \max (k, l)$ with $t_{i} \in\left(t_{k}, t_{l}\right)$. Then

$$
\begin{gathered}
\#\left\{n: J_{n}=\left[t_{k}, t_{l}\right]\right\} \leq 5, \\
\#\left\{n: J_{n} \subset\left[t_{k}, t_{l}\right] \text { and }\left|J_{n}\right|>\left|\left[t_{k}, t_{l}\right]\right| / 2\right\} \leq 25 .
\end{gathered}
$$

The following property of a general Franklin system has also been obtained in [10, Lemma 4.6]: 
Lemma 3.3. Let $\mathcal{T}$ be an admissible sequence of knots in $[0,1]$ with the corresponding Franklin system $\left\{f_{n}, n \geq 0\right\}$. There is a constant $C>0$, independent of $\mathcal{T}$, such that for each interval $V=[\alpha, \beta] \subset[0,1]$,

$$
\sum_{n: J_{n} \subset V}\left|J_{n}\right|^{1 / 2} \int_{V^{\mathrm{c}}}\left|f_{n}(t)\right| d t \leq C|V| .
$$

Now, we prove some additional properties of general Franklin systems needed in what follows.

LEMMA 3.4. Let $\mathcal{T}$ be an admissible sequence of knots in $[0,1]$ satisfying the strong regularity condition with parameter $\gamma \geq 1$. Let $\left\{f_{n}, n \geq 0\right\}$ be the corresponding Franklin system, with the corresponding family of J-intervals $\left\{J_{n}, n \geq 0\right\}$. For fixed $\Delta=I_{n, i}$ for some $n, i$, and $k \geq 0$, let

$$
N(\Delta, k)=\left\{n \geq 0: f_{n} \text { is linear on } \Delta \text { and } d_{n}(\Delta)=k\right\} .
$$

Then there is a constant $C_{\gamma}$, depending only on $\gamma$, such that

$$
\sum_{n \in N(\Delta, k)} \frac{\left|J_{n}\right|}{\left|J_{n}\right|+\operatorname{dist}\left(J_{n}, \Delta\right)+|\Delta|} \leq C_{\gamma}(k+1) .
$$

Proof. First, consider the case $k>0$. Then $J_{n} \subset \Delta^{\mathrm{c}}$ and the set $N(\Delta, k)$ splits into two subsets $N^{+}(\Delta, k), N^{-}(\Delta, k)$, according to the position of $J_{n}$ with respect to $\Delta: J_{n}$ is to the right of $\Delta$ for $n \in N^{+}(\Delta, k)$, and to the left of $\Delta$ for $n \in N^{-}(\Delta, k)$.

Consider first the case of $n \in N^{+}(\Delta, k)$. The corresponding intervals $J_{n}$ can be grouped into packets, with intervals in one packet having common left endpoint, and with maximal intervals from different packets disjoint. Let $J_{n}^{r}$ denote the right half of $J_{n}$. Note that

$$
\left|J_{n}\right|+\operatorname{dist}\left(J_{n}, \Delta\right)+|\Delta| \sim \operatorname{dist}(t, \Delta)+|\Delta| \quad \text { for } t \in J_{n}^{r},
$$

and by Lemma 3.2, each point belongs to at most 25 intervals $J_{n}^{r}$. In addition, as $d_{n}(\Delta)=k$, it follows by strong regularity of $\mathcal{T}$ that

$$
\left|J_{n}\right| \leq \gamma^{k}|\Delta| \text { and } \operatorname{dist}\left(J_{n}, \Delta\right) \leq C_{\gamma} \gamma^{k}|\Delta|,
$$

where $C_{\gamma}$ depends only on $\gamma$. Combining these facts we get

$$
\begin{aligned}
\sum_{n \in N^{+}(\Delta, k)} \frac{\left|J_{n}\right|}{\left|J_{n}\right|+\operatorname{dist}\left(J_{n}, \Delta\right)+|\Delta|} & \leq C \sum_{n \in N^{+}(\Delta, k)} \int_{J_{n}^{r}} \frac{1}{\operatorname{dist}(t, \Delta)+|\Delta|} d t \\
& \leq C \int_{|\Delta|}^{C_{\gamma} \gamma^{k}|\Delta|} \frac{1}{t} d t \\
& =C\left(\ln \left(C_{\gamma} \gamma^{k}|\Delta|\right)-\ln |\Delta|\right) \leq C_{\gamma} k .
\end{aligned}
$$

The other part $\sum_{n \in N^{-}(\Delta, k)} \ldots$ is treated analogously. 
The case $k=0$ is similar, but it also requires consideration of the case $J_{n}=\Delta$. However, by Lemma 3.2, the number of such $n$ 's does not exceed 5 .

LEMMA 3.5. Let $\mathcal{T}$ be an admissible sequence of knots in $[0,1]$ satisfying the strong regularity condition for pairs with parameter $\gamma \geq 1$.

(i) Let $\Lambda, \Gamma$ be two adjacent intervals of the partition $\pi_{n}$ such that $|\Lambda| \leq$ $\frac{1}{2 \gamma}|\Gamma|$, and let $s=\min \left\{i>n: t_{i} \in \Lambda \cup \Gamma\right\}$. Then $t_{s} \in \Gamma$. Moreover, if $\Gamma^{\prime}, \Gamma^{\prime \prime}$ is the splitting of $\Gamma$ by $t_{s}$ with $\Gamma^{\prime}$ adjacent to $\Lambda$, then $\left|\Gamma^{\prime}\right| \geq$ $\frac{1}{2 \gamma}|\Gamma|$.

(ii) Let $\Gamma, \Lambda, \Gamma^{\prime}, \Gamma^{\prime \prime}$ and $s$ be as in (i), and let $\Delta$ be the other neighbour of $\Gamma$ in $\pi_{s-1}$. Then $|\Delta| \leq \gamma|\Gamma|$, and consequently

$$
|\Lambda|+\left|\Gamma^{\prime}\right|,\left|\Gamma^{\prime}\right|+\left|\Gamma^{\prime \prime}\right|,\left|\Gamma^{\prime \prime}\right|+|\Delta| \sim_{\gamma}|\Gamma| .
$$

(iii) Let $V_{1} \supset V_{2} \supset V_{3} \supset V_{4}$ be four different intervals from the sequence of partitions corresponding to $\mathcal{T}$. Then

$$
\left|V_{4}\right| \leq \frac{2 \gamma}{2 \gamma+1}\left|V_{1}\right|
$$

Proof. To prove (i), note first that the assumption $|\Lambda| \leq \frac{1}{2 \gamma}|\Gamma|$ and the fact that the knots are at most double imply that $|\Gamma|>0$. Suppose that $t_{s} \in \Lambda$. Then $t_{s}$ splits $\Lambda$ into $\Lambda^{\prime}, \Lambda^{\prime \prime}$, with $\Lambda^{\prime}$ denoting the part adjacent to $\Gamma$. Then $\Lambda^{\prime}, \Lambda^{\prime \prime}, \Gamma$ are intervals of $\pi_{s}$, and moreover $\Lambda^{\prime}, \Lambda^{\prime \prime}$ and $\Lambda^{\prime}, \Gamma$ are neighbouring pairs. But then, by strong regularity for pairs,

$$
|\Gamma| \leq|\Gamma|+\left|\Lambda^{\prime}\right| \leq \gamma\left(\left|\Lambda^{\prime}\right|+\left|\Lambda^{\prime \prime}\right|\right)=\gamma|\Lambda| \leq|\Gamma| / 2
$$

which is impossible. Thus, $t_{s} \in \Gamma$, and it splits $\Gamma$ into intervals $\Gamma^{\prime}, \Gamma^{\prime \prime}$, with $\Gamma^{\prime}$ adjacent to $\Lambda$. Now, $\Lambda, \Gamma^{\prime}, \Gamma^{\prime \prime}$ are intervals of $\pi_{s}$, and $\left(\Lambda, \Gamma^{\prime}\right),\left(\Gamma^{\prime}, \Gamma^{\prime \prime}\right)$ are adjacent pairs. If $\left|\Gamma^{\prime}\right|<\frac{1}{2 \gamma}|\Gamma|$, then, by strong regularity for pairs,

$$
|\Gamma|=\left|\Gamma^{\prime}\right|+\left|\Gamma^{\prime \prime}\right| \leq \gamma\left(|\Lambda|+\left|\Gamma^{\prime}\right|\right)<|\Gamma|,
$$

which is impossible. This completes the proof of part (i).

To check (ii), note that $\Gamma^{\prime}, \Gamma^{\prime \prime}$ and $\Gamma^{\prime \prime}, \Delta$ are neighbouring pairs in $\pi_{s}$. Therefore, by strong regularity for pairs,

$$
\frac{1}{\gamma}\left(\left|\Gamma^{\prime \prime}\right|+|\Delta|\right) \leq|\Gamma|=\left|\Gamma^{\prime}\right|+\left|\Gamma^{\prime \prime}\right| \leq \gamma\left(\left|\Gamma^{\prime \prime}\right|+|\Delta|\right) .
$$

This, (i) and the assumption on $|\Lambda|$ give (ii).

Now, we turn to the proof of (iii). It is enough to consider the case when each $V_{i+1}$ is obtained by the first splitting of $V_{i}$ by a knot of $\mathcal{T}$.

Let $V_{1}^{\prime}, V_{1}^{\prime \prime}=V_{2}$ be the intervals obtained by the first splitting of $V_{1}$. If $\left|V_{2}\right| \leq \frac{2 \gamma}{2 \gamma+1}\left|V_{1}\right|$, then the result follows. If not, then we have $\left|V_{1}^{\prime}\right|<$ $\frac{1}{2 \gamma+1}\left|V_{1}\right|<\frac{1}{2 \gamma}\left|V_{2}\right|$. It follows by (i) that the first knot of $\mathcal{T}$ falling into $V_{1}^{\prime} \cup V_{2}$ 
must be in $V_{2}$ and if $V_{2}^{\prime}, V_{2}^{\prime \prime}$ are the intervals obtained by the first splitting of $V_{2}$, with $V_{2}^{\prime}$ being a neighbour of $V_{1}^{\prime}$, then $\left|V_{2}^{\prime}\right| \geq \frac{1}{2 \gamma}\left|V_{2}\right|$. Consequently, $\left|V_{2}^{\prime \prime}\right| \leq \frac{2 \gamma-1}{2 \gamma}\left|V_{2}\right| \leq \frac{2 \gamma}{2 \gamma+1}\left|V_{1}\right|$. Thus, if $V_{3}=V_{2}^{\prime \prime}$, then the result follows. It remains to consider the case $V_{3}=V_{2}^{\prime}$. If $\left|V_{1}^{\prime}\right| \geq \frac{1}{2 \gamma}\left|V_{3}\right|$ or $\left|V_{2}^{\prime \prime}\right| \geq \frac{1}{2 \gamma}\left|V_{3}\right|$, then $\left|V_{3}\right| \leq \frac{2 \gamma}{2 \gamma+1}\left|V_{1}\right|$, and the result follows. If not, the two neighbours of $V_{3}$ are $V_{1}^{\prime}$ and $V_{2}^{\prime \prime}$. Therefore, by (i), the first knot of $\mathcal{T}$ falling into $V_{1}^{\prime} \cup V_{3} \cup V_{2}^{\prime \prime}$ must be in $V_{3}$. Note that if $V_{3}^{\prime}, V_{3}^{\prime \prime}$ are the intervals obtained by the first splitting of $V_{3}$, then one of them is a neighbour of $V_{1}^{\prime}$, and the other is a neighbour of $V_{2}^{\prime \prime}$, so (i) also implies that $\left|V_{3}^{\prime}\right|,\left|V_{3}^{\prime \prime}\right| \geq \frac{1}{2 \gamma}\left|V_{3}\right|$. Consequently, $\left|V_{3}^{\prime}\right|,\left|V_{3}^{\prime \prime}\right| \leq \frac{2 \gamma-1}{2 \gamma}\left|V_{3}\right| \leq \frac{2 \gamma}{2 \gamma+1}\left|V_{1}\right|$. As $V_{4}$ is one of $V_{3}^{\prime}, V_{3}^{\prime \prime}$, this completes the proof of (iii).

3.2.1. Properties of orthogonal projections onto $S(\sigma)$. Partial sums with respect to a general Franklin system are orthogonal projections onto spaces of piecewise linear functions with corresponding knots. Therefore, we will need some properties of these orthogonal projections.

As above, let $\sigma$ be a (finite) sequence of at most double knots in $[0,1]$ and let $S(\sigma)$ be the space of piecewise linear functions with knots $\sigma$. Let $Q_{\sigma}$ be the orthogonal (in $L^{2}[0,1]$ ) projection onto $S(\sigma)$. We will need the following properties of $Q_{\sigma}$ :

Proposition 3.6. (i) Let $1 \leq p \leq \infty$ and $f \in L^{p}[0,1]$. Then $\left\|Q_{\sigma} f\right\|_{p}$ $\leq 3\|f\|_{p}$.

(ii) Let $f \in L^{1}[0,1]$. Then $\sup _{\sigma}\left|Q_{\sigma} f\right| \leq 64 \mathcal{M} f$, and if $t$ is a Lebesgue point of $f$ then $Q_{\sigma} f(t) \rightarrow f(t)$ as $|\sigma| \rightarrow 0$ (where $|\sigma|$ denotes the diameter of the partition $\sigma)$.

Part (i) of Proposition 3.6 comes from [4], and part (ii) from [5].

\section{SOME AUXILIARY RESULTS}

Let $\mathcal{T}$ be an admissible sequence of knots with the corresponding general Franklin system $\left\{f_{n}, n \geq 0\right\}$. For a sequence $a=\left(a_{n}, n \geq 0\right)$ of coefficients, let

$$
P(\cdot)=\left(\sum_{n=0}^{\infty} a_{n}^{2} f_{n}^{2}(\cdot)\right)^{1 / 2}, \quad S(\cdot)=\sup _{m \geq 0}\left|\sum_{n=0}^{m} a_{n} f_{n}(\cdot)\right| .
$$

If $f \in L^{1}[0,1]$, then we denote by $P f, S f$ the functions $P, S$ corresponding to $a_{n}=a_{n}(f)=\left(f, f_{n}\right)$.

Consider the following conditions:

(A) $\quad P \in L^{1}[0,1]$.

(B) The series $\sum_{n=0}^{\infty} a_{n} f_{n}$ converges unconditionally in $L^{1}[0,1]$. 
(C) $S \in L^{1}[0,1]$.

(D) There is $f \in H^{1}[0,1]$ such that $a_{n}=\left(f, f_{n}\right)$.

In this section we discuss the relations between these conditions under various regularity assumptions on $\mathcal{T}$.

Let us recall that in the case of the classical Franklin system (i.e. with dyadic knots), or of spline systems of higher order (also with dyadic knots), the relations (and equivalences) between these conditions have been studied in several papers (see e.g. [3], [15], [16], [8]), including also the case $p<1$. For higher order spline systems, the results are known for the range of $p$ depending on the order of splines (see [15], [16]), while for the classical Franklin system it is known that conditions (A), (B), (C) are equivalent for all $0<p \leq 1$ (see [8]). In [9], the equivalence of these conditions has been proved for quasi-dyadic strongly regular sequences of knots (also including the case $p<1)$.

Here, we study the mutual relations of these conditions under weaker assumptions, but only for $p=1$. The general schemes of proofs are similar to those in [8] and [9], but now we have to avoid arguments like (2.3), i.e. comparison of lengths of intervals which are far apart. This kind of argument is replaced by investigation of the geometry of the intervals $J_{n}$, similarly to $[10]$.

For the proofs below, the following known property of polynomials is needed:

FACT 4.1. Let $k \in \mathbb{N}$ and $0<\varrho<1$ be fixed. There is a constant $M=$ $M_{k, \varrho}$, depending only on $k$ and $\varrho$, such that for every interval $[a, b]$, set $A \subset[a, b]$ with $|A| \geq \varrho|[a, b]|$ and polynomial $Q$ of degree $k$,

$$
\max _{t \in[a, b]}|Q(t)| \leq M_{k, \varrho} \sup _{t \in A}|Q(t)| \quad \int_{a}^{b}|Q(t)| d t \leq M_{k, \varrho} \int_{A}|Q(t)| d t .
$$

Implication $(\mathrm{B}) \Rightarrow(\mathrm{A})$. The implication $(\mathrm{B}) \Rightarrow(\mathrm{A})$ is an immediate consequence of the Khinchin inequality. We formulate it just for general Franklin systems:

FACT 4.2. Let $\mathcal{T}$ be an admissible sequence of knots with the corresponding general Franklin system $\left\{f_{n}, n \geq 0\right\}$, and let $a=\left(a_{n}, n \geq 0\right)$ be a sequence of coefficients. If the series $\sum_{n=0}^{\infty} a_{n} f_{n}$ converges unconditionally in $L^{1}[0,1]$, then $P \in L^{1}[0,1]$. Moreover, there is a constant $C>0$, independent of $\mathcal{T}$ and $a$, such that

$$
\|P\|_{1} \leq C \sup _{\varepsilon=\left(\varepsilon_{n}\right)_{n \geq 0}, \varepsilon_{n}= \pm 1}\left\|\sum_{n=0}^{\infty} \varepsilon_{n} a_{n} f_{n}\right\|_{1} .
$$

Implications $(\mathrm{A}) \Rightarrow(\mathrm{B})$ and $(\mathrm{A}) \Rightarrow(\mathrm{C})$. We show that these implications hold for any admissible sequence $\mathcal{T}$. 
Proposition 4.3. Let $\mathcal{T}$ be an admissible sequence of knots with the corresponding general Franklin system $\left\{f_{n}, n \geq 0\right\}$, and let $a=\left(a_{n}, n \geq 0\right)$ be a sequence of coefficients such that $P \in L^{1}[0,1]$. Then $S \in L^{1}[0,1]$ and the series $\sum_{n=0}^{\infty} a_{n} f_{n}$ converges unconditionally in $L^{1}[0,1]$. Moreover, there is a constant $C>0$, independent of $\mathcal{T}$ and a, such that

$$
\|S\|_{1} \leq C\|P\|_{1}, \quad \sup _{\varepsilon=\left(\varepsilon_{n}\right)_{n \geq 0}, \varepsilon_{n}= \pm 1}\left\|\sum_{n=0}^{\infty} \varepsilon_{n} a_{n} f_{n}\right\|_{1} \leq C\|P\|_{1} .
$$

Proof. For convenience, assume $\|P\|_{1}=1$. Define

$$
\mathcal{I}_{\mathcal{T}}=\bigcup_{n \geq 1}\left\{I_{n, i}: 1 \leq i \leq n\right\}
$$

where $I_{n, i}$ are as in $(2.2)$, and $\mathcal{M}_{\mathcal{T}}(f, t)=\sup _{t \in \operatorname{int} I_{n, i}}\left(1 /\left|I_{n, i}\right|\right) \int_{I_{n, i}}|f(u)| d u$. Let $E_{0}=B_{0}=[0,1]$, and for $r \geq 1$,

$$
\begin{aligned}
& E_{r}=\left\{t \in[0,1]: \sum_{n=0}^{\infty} a_{n}^{2} f_{n}^{2}(t)>2^{r}\right\}, \\
& B_{r}=\left\{t \in[0,1]: \mathcal{M}_{\mathcal{T}}\left(\chi_{E_{r}}, t\right)>1 / 2\right\} .
\end{aligned}
$$

Then

$$
B_{r}=\bigcup_{I \in \mathcal{I}_{r}} I
$$

where $\mathcal{I}_{r}$ is the collection of maximal intervals of $\mathcal{I}_{\mathcal{T}}$ included in $B_{r}$. Further, for $I \in \mathcal{I}_{r}$ let

$$
\psi_{I}=\sum_{n: J_{n} \subset I, J_{n} \not \subset B_{r+1}} a_{n} f_{n} .
$$

Since $J_{n} \not \subset B_{r+1}$, we have $\left|E_{r+1}^{\mathrm{c}} \cap J_{n}\right| \geq\left|J_{n}\right| / 2$, so by Fact 4.1 and (3.6) for $p=2$,

$$
\int_{E_{r+1}^{\mathrm{c}} \cap J_{n}} f_{n}^{2}(t) d t \geq C \int_{J_{n}} f_{n}^{2}(t) d t \geq C .
$$

Therefore

$$
\begin{aligned}
\left\|\psi_{I}\right\|_{2}^{2} & =\sum_{n: J_{n} \subset I, J_{n} \not \subset B_{r+1}} a_{n}^{2} \leq C \sum_{n: J_{n} \subset I, J_{n} \not \subset B_{r+1}} a_{n}^{2} \int_{E_{r+1}^{\mathrm{c}} \cap J_{n}} f_{n}^{2}(t) d t \\
& \leq \int_{I \cap E_{r+1}^{\mathrm{c}}} \sum_{n: J_{n} \subset I, J_{n} \not \subset B_{r+1}} a_{n}^{2} f_{n}^{2}(t) d t \leq C 2^{r}|I| .
\end{aligned}
$$

The rest of the proof is analogous to the proof of Lemma 4.6 of [9] or Theorem 1.1 (sufficiency part) in [8], but we present it for completeness.

For $\varepsilon=\left(\varepsilon_{n}, n \geq 0\right)$ with $\varepsilon_{n} \in\{-1,1\}$ and $I \in \mathcal{I}_{r}$, let

$$
\psi_{I, \varepsilon}=\sum_{n: J_{n} \subset I, J_{n} \not \subset B_{r+1}} \varepsilon_{n} a_{n} f_{n} .
$$


The series defining $\psi_{I, \varepsilon}$ converges in $L^{2}[0,1]$, so also in $L^{1}[0,1]$. Therefore, to prove the unconditional convergence of $\sum_{n=0}^{\infty} a_{n} f_{n}$ in $L^{1}[0,1]$, it is enough to show that

$$
\sum_{r=0}^{\infty} \sum_{I \in \mathcal{I}_{r}}\left\|\psi_{I, \varepsilon}\right\|_{1} \leq C .
$$

For this, we need to estimate $\left\|\psi_{I, \varepsilon}\right\|_{1}$. First, by the Cauchy-Schwarz inequality we have

$$
\int_{I}\left|\psi_{I, \varepsilon}(t)\right| d t \leq\left\|\psi_{I, \varepsilon}\right\|_{2} \cdot\left\|\chi_{I}\right\|_{2} \leq C 2^{r / 2}|I| .
$$

To estimate the integral over $I^{\mathrm{c}}$, note that if $J_{n} \not \subset B_{r+1}$, then $\left|a_{n}\right| \leq$ $C 2^{r / 2}\left|J_{n}\right|^{1 / 2}$. Indeed, $f_{n}$ is linear on $J_{n}$, and $\sup _{J_{n}}\left|f_{n}\right| \sim\left|J_{n}\right|^{-1 / 2}$ (cf. (3.6)), so by Fact 4.1 there is $C>0$ such that $\left|f_{n}\right| \geq C\left|J_{n}\right|^{-1 / 2}$ on a subset $V \subset J_{n}$ with $|V|>\left|J_{n}\right| / 2$. If $\left|a_{n}\right|>(1 / C) 2^{(r+1) / 2}\left|J_{n}\right|^{1 / 2}$, then $\left|a_{n} f_{n}\right|>2^{(r+1) / 2}$ on $V$, which implies $P^{2}>2^{r+1}$ on $V$, and consequently $J_{n} \subset B_{r+1}$, contrary to the choice of $J_{n}$. Now, using this estimate for $a_{n}$ and Lemma 3.3 we get

$$
\begin{aligned}
\int_{I^{\mathrm{c}}}\left|\psi_{I, \varepsilon}(t)\right| d t & \leq \sum_{n: J_{n} \subset I, J_{n} \not \subset B_{r+1}}\left|a_{n}\right| \int_{I^{\mathrm{c}}}\left|f_{n}(t)\right| d t \\
& \leq C 2^{r / 2} \sum_{n: J_{n} \subset I, J_{n} \not \subset B_{r+1}}\left|J_{n}\right|^{1 / 2} \int_{I^{\mathrm{c}}}\left|f_{n}(t)\right| d t \leq C 2^{r / 2}|I| .
\end{aligned}
$$

Combining the last inequality with (4.1) we find $\left\|\psi_{I, \varepsilon}\right\|_{1} \leq C 2^{r / 2}|I|$. This implies that

$$
\begin{aligned}
\sum_{r=0}^{\infty} \sum_{I \in \mathcal{I}_{r}}\left\|\psi_{I, \varepsilon}\right\|_{1} & \leq C \sum_{r=0}^{\infty} \sum_{I \in \mathcal{I}_{r}} 2^{r / 2}|I| \leq C \sum_{r=0}^{\infty} 2^{r / 2}\left|B_{r}\right| \\
& \leq C \sum_{r=0}^{\infty} 2^{r / 2}\left|E_{r}\right| \leq C\|P\|_{1}=C,
\end{aligned}
$$

yielding both the unconditional convergence of $\sum_{n=0}^{\infty} a_{n} f_{n}$ in $L^{1}[0,1]$ and the estimate

$$
\sup _{\varepsilon}\left\|\sum_{n=0}^{\infty} \varepsilon_{n} a_{n} f_{n}\right\|_{1} \leq C .
$$

It remains to estimate $\|S\|_{1}$. Clearly, $\|S\|_{1} \leq \sum_{r=0}^{\infty} \sum_{I \in \mathcal{I}_{r}}\left\|S \psi_{I}\right\|_{1}$, and $S \psi_{I} \leq \sum_{n: J_{n} \subset I, J_{n} \not \subset B_{r+1}}\left|a_{n} f_{n}\right|$. Moreover, it follows from Proposition 3.6(ii) and (2,2)-type of $\mathcal{M}$ that $\left\|S \psi_{I}\right\|_{2} \leq C\left\|\mathcal{M} \psi_{I}\right\|_{2} \leq C\left\|\psi_{I}\right\|_{2}$. Therefore, splitting $\|S\|_{1}$ into $\int_{I} \ldots$ and $\int_{I^{c}} \ldots$, and treating each part as the corresponding part of the estimate for $\psi_{I, \varepsilon}$ we find $\left\|S \psi_{I}\right\|_{1} \leq C 2^{r / 2}|I|$, where $I \in \mathcal{I}_{r}$. Finally, summing over $r \geq 0$ and $I \in \mathcal{I}_{r}$ we get $\|S\|_{1} \leq C$. 
Implications $(\mathrm{D}) \Rightarrow(\mathrm{A})$ and $(\mathrm{D}) \Rightarrow(\mathrm{C})$. We show that these implications hold for strongly regular sequences $\mathcal{T}$.

It is enough to prove $(D) \Rightarrow(A)$, since then $(D) \Rightarrow(C)$ follows by Proposition 4.3. However, the direct proof of $(\mathrm{D}) \Rightarrow(\mathrm{C})$ uses the same arguments as the proof of $(\mathrm{D}) \Rightarrow(\mathrm{A})$, so we present them together.

LEMMA 4.4. Let $\mathcal{T}$ be an admissible sequence of knots with the corresponding general Franklin system $\left\{f_{n}, n \geq 0\right\}$. Let $\gamma \geq 1$ and assume that $\mathcal{T}$ satisfies the strong regularity condition with parameter $\gamma$. Then there is a constant $C_{\gamma}$, depending only on $\gamma$, such that for each atom $\phi$,

$$
\|S \phi\|_{1} \leq C_{\gamma}, \quad\|P \phi\|_{1} \leq C_{\gamma} .
$$

Proof. Clearly, the estimates hold for $\phi \equiv 1$. Now, let $\phi$ be an atom such that $\int_{0}^{1} \phi(u) d u=0$, and let $\Gamma$ be an interval such that supp $\phi \subset \Gamma$, $\Gamma=[\alpha, \beta], \sup |\phi| \leq 1 /|\Gamma|$. Write $a_{n}=a_{n}(\phi)=\left(\phi, f_{n}\right)$. Let $n_{\Gamma}=\max \{n$ : $\left.\#\left(\pi_{n} \cap \Gamma\right) \leq 1\right\}$ and

$$
\begin{gathered}
P_{1} \phi=\left(\sum_{n=0}^{n_{\Gamma}} a_{n}^{2} f_{n}^{2}\right)^{1 / 2}, \quad P_{2} \phi=\left(\sum_{n=n_{\Gamma}+1}^{\infty} a_{n}^{2} f_{n}^{2}\right)^{1 / 2}, \\
S_{1} \phi=\max _{0 \leq m \leq n_{\Gamma}}\left|\sum_{n=0}^{m} a_{n} f_{n}\right|, \quad S_{2} \phi=\sup _{m \geq n_{\Gamma}+1}\left|\sum_{n=n_{\Gamma}+1}^{m} a_{n} f_{n}\right| .
\end{gathered}
$$

It is enough to show that

$$
\left\|P_{1} \phi\right\|_{1},\left\|P_{2} \phi\right\|_{1},\left\|S_{1} \phi\right\|_{1},\left\|S_{2} \phi\right\|_{1} \leq C_{\gamma} .
$$

First, we show that

$$
\sum_{n \leq n_{\Gamma}}\left|a_{n}\right| \cdot\left\|f_{n}\right\|_{1} \leq C_{\gamma}
$$

Indeed, for $n \leq n_{\Gamma}$, let $\Gamma_{n, \alpha}$ and $\Gamma_{n, \beta}$ be the intervals of linearity of $f_{n}$ containing $\alpha$ and $\beta$, respectively (for some $n$, these intervals may coincide). As $\Gamma \subset \Gamma_{n, \alpha} \cup \Gamma_{n, \beta}$, strong regularity with parameter $\gamma$ implies that

$$
\left|\Gamma_{n, \alpha}\right|,\left|\Gamma_{n, \beta}\right| \geq \frac{1}{\gamma+1}|\Gamma|
$$

Observe that by linearity of $f_{n}$ on $\Gamma_{n, \alpha}$ and $\Gamma_{n, \beta}$, and by (3.7) of Proposition 3.1 , for $\rho=\alpha, \beta$ we have

$$
\left|f_{n}^{\prime}\right|=\eta_{n, \rho} \leq C\left(\frac{2}{3}\right)^{d_{n}(\Gamma)} \frac{\left|J_{n}\right|^{1 / 2}}{\left|J_{n}\right|+\operatorname{dist}\left(J_{n}, \Gamma_{n, \rho}\right)+\left|\Gamma_{n, \rho}\right|} \cdot \frac{1}{\left|\Gamma_{n, \rho}\right|} \quad \text { on } \Gamma_{n, \rho} .
$$

Let $\tau \in \pi_{n_{\Gamma}} \cap \Gamma$. As $\int_{\Gamma} \phi(t) d t=0$, we get 


$$
\begin{aligned}
\left|a_{n}\right| & =\left|\int_{\Gamma} \phi(t)\left(f_{n}(t)-f_{n}(\tau)\right) d t\right| \\
& \leq \int_{\Gamma_{n, \alpha} \cap \Gamma} \frac{\eta_{n, \alpha} \cdot|t-\tau|}{|\Gamma|} d t+\int_{\Gamma_{n, \beta} \cap \Gamma} \frac{\eta_{n, \beta} \cdot|t-\tau|}{|\Gamma|} d t \leq C\left(a_{n, \alpha}+a_{n, \beta}\right),
\end{aligned}
$$

where

$$
a_{n, \rho}=\left(\frac{2}{3}\right)^{d_{n}(\Gamma)} \frac{\left|J_{n}\right|^{1 / 2}}{\left|J_{n}\right|+\operatorname{dist}\left(J_{n}, \Gamma_{n, \rho}\right)+\left|\Gamma_{n, \rho}\right|} \cdot \frac{|\Gamma|}{\left|\Gamma_{n, \rho}\right|}, \quad \rho=\alpha, \beta .
$$

Let us treat the case of $a_{n, \alpha}$; the case of $a_{n, \beta}$ is analogous. Let $\Delta_{1} \supset \cdots \supset \Delta_{s}$ be the collection of all different intervals appearing as $\Gamma_{n, \alpha}$ for $n \leq n_{\Gamma}$. By strong regularity,

$$
\frac{1}{\gamma+1}\left|\Delta_{i}\right| \leq\left|\Delta_{i+1}\right| \leq \frac{\gamma}{\gamma+1}\left|\Delta_{i}\right|
$$

Now, fix $\Delta_{i}$ and $k \geq 0$, and consider $n$ such that $\Gamma_{n, \alpha}=\Delta_{i}$ and $d_{n}(\Gamma)=k$. As there is at most one knot of $\pi_{n}$ in $\Gamma$, we have $\left|d_{n}(\Gamma)-d_{n}\left(\Delta_{i}\right)\right| \leq 1$. In addition, by the definition of $a_{n, \alpha}$ and the estimates of norms in Proposition 3.1 (cf. (3.4)) we have

$$
a_{n, \alpha}\left\|f_{n}\right\|_{1} \leq C\left(\frac{2}{3}\right)^{k} \frac{\left|J_{n}\right|}{\left|J_{n}\right|+\operatorname{dist}\left(J_{n}, \Delta_{i}\right)+\left|\Delta_{i}\right|} \cdot \frac{|\Gamma|}{\left|\Delta_{i}\right|} .
$$

Now, it follows from Lemma 3.4 that

$$
\sum_{n: \Gamma_{n, \alpha}=\Delta_{i}, d_{n}(\Gamma)=k} a_{n, \alpha}\left\|f_{n}\right\|_{1} \leq C_{\gamma}(k+1)\left(\frac{2}{3}\right)^{k} \frac{|\Gamma|}{\left|\Delta_{i}\right|} .
$$

Using the last inequality, (4.5) and (4.4) we get

$$
\begin{aligned}
\sum_{n \leq n_{\Gamma}} a_{n, \alpha}\left\|f_{n}\right\|_{1} & =\sum_{i=1}^{s} \sum_{k \geq 0} \sum_{n: \Gamma_{n, \alpha}=\Delta_{i}, d_{n}(\Gamma)=k} a_{n, \alpha}\left\|f_{n}\right\|_{1} \\
& \leq C_{\gamma} \sum_{i=1}^{s} \frac{|\Gamma|}{\left|\Delta_{i}\right|} \sum_{k \geq 0}(k+1)\left(\frac{2}{3}\right)^{k} \\
& \leq C_{\gamma} \frac{|\Gamma|}{\left|\Delta_{s}\right|} \leq C_{\gamma} .
\end{aligned}
$$

The part of the sum corresponding to $a_{n, \beta}$ is treated analogously, so we get (4.3).

It follows from (4.3) that

$$
\left\|P_{1} \phi\right\|_{1},\left\|S_{1} \phi\right\|_{1} \leq C_{\gamma} .
$$

Now, we turn to estimating $\left\|P_{2} \phi\right\|_{1}$ and $\left\|S_{2} \phi\right\|_{1}$. Consider the partition $\pi_{n_{\Gamma}+1}$. By the definition of $n_{\Gamma}$, there are exactly two knots of $\pi_{n_{\Gamma}+1}$ in $\Gamma$. To simplify the notation, let $\pi_{n_{\Gamma}+1}=\left\{0=\tau_{0}<\tau_{1}<\cdots<\tau_{n_{\Gamma}+1}=1\right\}$, 
and let $j$ be such that $\tau_{j}, \tau_{j+1} \in \Gamma$. Define further

$$
\begin{gathered}
V_{0}=\left[\tau_{j}, \tau_{j+1}\right], \quad V=\left[\tau_{j-1}, \tau_{j+2}\right] \\
V^{-}=\left[\tau_{j-1}, \tau_{j}\right], \quad V^{+}=\left[\tau_{j+1}, \tau_{j+2}\right], \quad \widetilde{V}=\left[\tau_{j-3}, \tau_{j+4}\right] .
\end{gathered}
$$

As $V_{0} \subset \Gamma \subset V$, it follows from the strong regularity of $\mathcal{T}$ that

$$
\left|V_{0}\right|,\left|V^{-}\right|,|V|,\left|V^{+}\right|,|\widetilde{V}| \sim_{\gamma}|\Gamma| .
$$

Observe that $\|\phi\|_{2} \leq|\Gamma|^{-1 / 2}$. Therefore, using (4.7) and the Cauchy-Schwarz inequality we get

$$
\int_{\widetilde{V}} P_{2} \phi(t) d t \leq\left\|\chi_{\tilde{V}}\right\|_{2} \cdot\|\phi\|_{2} \leq \frac{|\widetilde{V}|^{1 / 2}}{|\Gamma|^{1 / 2}} \leq C_{\gamma} .
$$

It follows by Proposition 3.6(ii) that $S_{2} \phi \leq 128 \mathcal{M} \phi$. Since $\mathcal{M}$ is of type $(2,2)$, by an analogous argument we find

$$
\int_{\widetilde{V}} S_{2} \phi(t) d t \leq C_{\gamma}
$$

It remains to estimate $\int_{\widetilde{V}^{\mathrm{c}}} P_{2} \phi(t) d t$ and $\int_{\widetilde{V}^{\mathrm{c}}} S_{2} \phi(t) d t$. To this end, it is sufficient to show that

$$
\sum_{n=n_{\Gamma}+1}^{\infty}\left|a_{n}\right| \int_{\tilde{V}^{c}}\left|f_{n}(t)\right| d t \leq C_{\gamma} .
$$

For each $n>n_{\Gamma}$, the endpoints of $\widetilde{V}$ are knots of $\pi_{n}$, so there are three possible positions of $J_{n}$ with respect to $\widetilde{V}: J_{n} \subset \widetilde{V}$, or $J_{n}$ is to the right of $\widetilde{V}$, or $J_{n}$ is to the left of $\widetilde{V}$.

If $J_{n} \subset \widetilde{V}$, then by (3.4) and the fact that $\phi$ is an atom,

$$
\left|a_{n}\right|=\left|\int_{\Gamma} \phi(t) f_{n}(t) d t\right| \leq \frac{\left\|f_{n}\right\|_{1}}{|\Gamma|} \leq C \frac{\left|J_{n}\right|^{1 / 2}}{|\Gamma|} .
$$

Therefore, applying Lemma 3.3 to $\widetilde{V}$ and using (4.7) we get

$$
\sum_{n: J_{n} \subset \widetilde{V}}\left|a_{n}\right| \int_{\widetilde{V}^{\mathrm{c}}}\left|f_{n}(t)\right| d t \leq C \frac{|\widetilde{V}|}{|\Gamma|} \leq C_{\gamma} .
$$

Now, let $J_{n}$ be to the right of $\widetilde{V}$. Denote by $\beta^{\prime}$ the right endpoint of $V$, and by $L_{n}$ the interval of linearity of $f_{n}$ with right endpoint $\beta^{\prime}$. By the choice of $\widetilde{V}$, for each $n>n_{\Gamma}$ there is at least one knot of $\pi_{n}$ between $\beta^{\prime}$ and the right endpoint of $\widetilde{V}$. Since $J_{n}$ is to the right of $\widetilde{V}$, this guarantees that $\beta^{\prime} \leq t_{n}^{-}$. Since $\Gamma \subset V$, the estimates of Proposition 3.1(a) and (3.7) yield

$$
\begin{aligned}
\left|a_{n}\right| & \leq \frac{1}{|\Gamma|} \int_{\Gamma}\left|f_{n}(t)\right| d t \leq \frac{C}{|\Gamma|} \int_{L_{n}}\left|f_{n}(t)\right| d t \\
& \leq C\left(\frac{2}{3}\right)^{d_{n}\left(\beta^{\prime}\right)} \frac{\left|J_{n}\right|^{1 / 2}}{\left|J_{n}\right|+\operatorname{dist}\left(J_{n}, L_{n}\right)+\left|L_{n}\right|} \cdot \frac{\left|L_{n}\right|}{|\Gamma|} .
\end{aligned}
$$


Let $\Lambda_{1} \supset \Lambda_{2} \supset \cdots$ be the collection of all different intervals appearing as $L_{n}$. Observe that $\Lambda_{1}=V^{+}$. Fix $\Lambda_{i}$ and $k \geq 0$, and consider $n$ such that $L_{n}=\Lambda_{i}$ and $d_{n}\left(\beta^{\prime}\right)=k$. Then (cf. (3.4) for the estimate of $\left\|f_{n}\right\|_{1}$ )

$$
\left|a_{n}\right| \int_{\widetilde{V}^{\mathrm{c}}}\left|f_{n}(t)\right| d t \leq\left|a_{n}\right| \cdot\left\|f_{n}\right\|_{1} \leq C\left(\frac{2}{3}\right)^{k} \frac{\left|J_{n}\right|}{\left|J_{n}\right|+\operatorname{dist}\left(J_{n}, \Lambda_{i}\right)+\left|\Lambda_{i}\right|} \cdot \frac{\left|\Lambda_{i}\right|}{|\Gamma|} .
$$

Applying Lemma 3.4 we get

$$
\sum_{n: L_{n}=\Lambda_{i}, d_{n}\left(\beta^{\prime}\right)=k}\left|a_{n}\right| \int_{\widetilde{V}^{c}}\left|f_{n}(t)\right| d t \leq C_{\gamma}\left(\frac{2}{3}\right)^{k}(k+1) \frac{\left|\Lambda_{i}\right|}{|\Gamma|} .
$$

Strong regularity yields

$$
\frac{1}{\gamma+1}\left|\Lambda_{i}\right| \leq\left|\Lambda_{i+1}\right| \leq \frac{\gamma}{\gamma+1}\left|\Lambda_{i}\right|
$$

As $\left|\Lambda_{1}\right| \sim_{\gamma}|\Gamma|$, by summing (4.12) over $i$ and $k$ we get

$$
\begin{aligned}
\sum_{n: J_{n} \text { to the right of } \widetilde{V}}\left|a_{n}\right| \int_{\widetilde{V}^{c}}\left|f_{n}(t)\right| d t & \leq C_{\gamma} \sum_{i=1}^{\infty} \frac{\left|\Lambda_{i}\right|}{|\Gamma|} \sum_{k=0}^{\infty}(k+1)\left(\frac{2}{3}\right)^{k} \\
& \leq C_{\gamma} \frac{\left|\Lambda_{1}\right|}{|\Gamma|} \leq C_{\gamma}
\end{aligned}
$$

The case of $J_{n}$ to the left of $\widetilde{V}$ is treated analogously. Putting together these cases and (4.11) we get (4.10). This completes the proof of Lemma 4.4.

As an immediate consequence of Lemma 4.4 we get the following:

Proposition 4.5. Let $\mathcal{T}$ be an admissible sequence of knots with the corresponding general Franklin system $\left\{f_{n}, n \geq 0\right\}$. Let $\gamma \geq 1$ and assume that $\mathcal{T}$ satisfies the strong regularity condition with parameter $\gamma$. Then there is a constant $C_{\gamma}$, depending only on $\gamma$, such that for each $f \in H^{1}[0,1]$,

$$
\|S f\|_{1} \leq C_{\gamma}\|f\|_{H^{1}}, \quad\|P f\|_{1} \leq C_{\gamma}\|f\|_{H^{1}} .
$$

The next proposition indicates that the above result cannot be extended to arbitrary partitions.

Proposition 4.6. Let $\mathcal{T}$ be an admissible sequence of knots from $[0,1]$ satisfying the strong regularity condition for pairs with parameter $\gamma$, but not satisfying any strong regularity condition. Let $\left\{f_{n}, n \geq 0\right\}$ be the corresponding Franklin system. Then

$$
\sup \left\|\sup _{n \geq 0}\left|a_{n}(\phi) f_{n}\right|\right\|_{1}=\infty,
$$

where the supremum is taken over all atoms $\phi$, and $a_{n}(\phi)=\left(\phi, f_{n}\right)$. 
Proof. Fix $k>0$. As $\mathcal{T}$ is not strongly regular, there are $n_{0}>0$ and two neighbouring intervals $\Lambda, \Gamma_{0}$ of the partition $\pi_{n_{0}}$ such that

$$
|\Lambda| \leq\left(\frac{1}{4 \gamma}\right)^{k+\varrho_{\gamma}}\left|\Gamma_{0}\right|
$$

where the choice of $\varrho_{\gamma} \geq 1$ will be explained later. First, consider the case when $\Lambda$ is a non-degenerate interval, i.e. $|\Lambda|>0$. For convenience, assume that the common endpoint $\tau$ of $\Lambda$ and $\Gamma_{0}$ is the right endpoint of $\Lambda$ and left endpoint of $\Gamma_{0}$. Consider the function

$$
\phi=\frac{1}{2|\Lambda|}\left(\chi_{\Lambda}-\chi_{[\tau, \tau+|\Lambda|]}\right) .
$$

Clearly, it is an atom. Let

$$
n_{1}=\min \left\{n>n_{0}: t_{n} \in \Lambda \cup \Gamma_{0} \text { and } t_{n} \text { is not an endpoint of } \Gamma_{0}\right\} .
$$

Next, we will find a pair of disjoint intervals $\Gamma_{1}, L_{1} \subset \Gamma_{0}$ such that $\Lambda, \Gamma_{1}$ are neighbouring intervals of some partition $\pi_{l}$ with $l \geq n_{1}$, and

$$
|\Lambda| \leq\left(\frac{1}{4 \gamma}\right)^{k-1+\varrho_{\gamma}}\left|\Gamma_{1}\right|, \quad \int_{L_{1}}\left|a_{n_{1}}(\phi) f_{n_{1}}(t)\right| d t \sim_{\gamma} 1 .
$$

It follows from Lemma 3.5(i) (note that by the strong regularity for pairs, in our situation only the right endpoint of $\Gamma_{0}$ may be a double knot in some $\pi_{n}$ with $\left.n_{0}<n \leq n_{1}\right)$ that $t_{n_{1}} \in \Gamma_{0}$, and for $\Gamma_{0}^{\prime}=\left[\tau, t_{n_{1}}\right]$ we have $\left|\Gamma_{0}^{\prime}\right| \geq \frac{1}{2 \gamma}\left|\Gamma_{0}\right|$. Note that both $t_{n_{1}}$ and $\tau=t_{n_{1}}^{-}$are simple knots in $\pi_{n_{1}}$, and moreover the right endpoint of $\Gamma_{0}$ is $t_{n_{1}}^{+}$. To simplify the notation, we assume that the right endpoint of $\Gamma_{0}$ is also a simple knot of $\pi_{n_{1}}$; the case when it is a double knot is similar, but instead of $f_{n_{1}}\left(t_{n_{1}}^{+}\right)$we use $\lim _{t \rightarrow t_{n_{1}}-0} f_{n_{1}}(t)$.

Then by Lemma 3.5(ii) and (3.4) of Proposition 3.1,

$$
\left|f_{n_{1}}\left(t_{n_{1}}\right)\right|,\left|f_{n_{1}}\left(t_{n_{1}}^{+}\right)\right|,\left|f_{n_{1}}(\tau)\right| \sim_{\gamma}\left|\Gamma_{0}\right|^{-1 / 2}
$$

These estimates, the sign changes of $f_{n}$ (cf. Proposition 3.1) and piecewise linearity of $f_{n}$ imply that, denoting by $\zeta_{n_{1}}, \eta_{n_{1}}$ the values of $f_{n_{1}}^{\prime}$ on $\Lambda$ and $\Gamma_{0}^{\prime}$, respectively, we have

$$
\left|\zeta_{n_{1}}\right| \sim_{\gamma} \frac{\left|\Gamma_{0}\right|^{-1 / 2}}{|\Lambda|}, \quad\left|\eta_{n_{1}}\right| \sim_{\gamma} \frac{\left|\Gamma_{0}\right|^{-1 / 2}}{\left|\Gamma_{0}\right|} .
$$

Denoting by $C_{\gamma} \geq c_{\gamma}$ the constants from these equivalences we have

$$
\begin{aligned}
\left|\zeta_{n_{1}}+\eta_{n_{1}}\right| & \leq\left|\zeta_{n_{1}}\right|+\left|\eta_{n_{1}}\right| \\
& \leq C_{\gamma} \frac{\left|\Gamma_{0}\right|^{-1 / 2}}{|\Lambda|}\left(1+\frac{|\Lambda|}{\left|\Gamma_{0}\right|}\right) \leq C_{\gamma} \frac{\left|\Gamma_{0}\right|^{-1 / 2}}{|\Lambda|}\left(1+\frac{1}{(4 \gamma)^{\varrho_{\gamma}}}\right), \\
\left|\zeta_{n_{1}}+\eta_{n_{1}}\right| & \geq\left|\zeta_{n_{1}}\right|-\left|\eta_{n_{1}}\right| \\
& \geq \frac{\left|\Gamma_{0}\right|^{-1 / 2}}{|\Lambda|}\left(c_{\gamma}-C_{\gamma} \frac{|\Lambda|}{\left|\Gamma_{0}\right|}\right) \geq \frac{\left|\Gamma_{0}\right|^{-1 / 2}}{|\Lambda|}\left(c_{\gamma}-\frac{C_{\gamma}}{(4 \gamma)^{\varrho_{\gamma}}}\right) .
\end{aligned}
$$


Now, we fix $\varrho_{\gamma} \geq 1$ large enough to guarantee $c_{\gamma}-C_{\gamma} /(4 \gamma)^{\varrho_{\gamma}} \geq c_{\gamma} / 2$. It should be clear that $\varrho_{\gamma}$ depends only on $\gamma$.

Let us estimate $a_{n_{1}}(\phi)$. Since $|\Lambda|<\left|\Gamma_{0}^{\prime}\right|$, we have $\operatorname{supp} \phi \subset \Lambda \cup \Gamma_{0}^{\prime}$, and

$$
\begin{aligned}
\left|a_{n_{1}}(\phi)\right| & =\left|\int_{0}^{1} \phi(t) f_{n_{1}}(t) d t\right|=\left|\int_{0}^{1} \phi(t)\left(f_{n_{1}}(t)-f_{n_{1}}(\tau)\right) d t\right| \\
& =\frac{1}{2|\Lambda|}\left|\int_{\tau-|\Lambda|}^{\tau} \zeta_{n_{1}} \cdot(t-\tau) d t-\int_{\tau}^{\tau+|\Lambda|} \eta_{n_{1}} \cdot(t-\tau) d t\right| \\
& =\frac{1}{4}|\Lambda|\left|\zeta_{n_{1}}+\eta_{n_{1}}\right| \sim_{\gamma}\left|\Gamma_{0}\right|^{-1 / 2} .
\end{aligned}
$$

This and (4.15) imply that

$$
\left|a_{n_{1}}(\phi) f_{n_{1}}\left(t_{n_{1}}\right)\right|,\left|a_{n_{1}}(\phi) f_{n_{1}}(\tau)\right|,\left|a_{n_{1}}(\phi) f_{n_{1}}\left(t_{n_{1}}^{+}\right)\right| \sim_{\gamma} 1 /\left|\Gamma_{0}\right| .
$$

If $\left|\Gamma_{0}^{\prime \prime}\right| \geq \frac{1}{2 \gamma}\left|\Gamma_{0}^{\prime}\right|$, then we put $L_{1}=\Gamma_{0}^{\prime \prime}=\left[t_{n_{1}}, t_{n_{1}}^{+}\right]$and $\Gamma_{1}=\Gamma_{0}^{\prime}=\left[\tau, t_{n_{1}}\right]$ : in this case, we have $\left|\Gamma_{1}\right| \geq \frac{1}{2 \gamma}\left|\Gamma_{0}\right|$, which gives

$$
|\Lambda| \leq\left(\frac{1}{4 \gamma}\right)^{k-1+\varrho_{\gamma}} \cdot \frac{1}{4 \gamma}\left|\Gamma_{0}\right| \leq\left(\frac{1}{4 \gamma}\right)^{k-1+\varrho_{\gamma}}\left|\Gamma_{1}\right| .
$$

Moreover, it follows from (4.16) (cf. Fact 4.1) that $\int_{L_{1}}\left|a_{n_{1}}(\phi) f_{n_{1}}(t)\right| d t \sim_{\gamma} 1$. So in this case $L_{1}, \Gamma_{1}$ satisfy (4.14).

If $\left|\Gamma_{0}^{\prime \prime}\right| \leq \frac{1}{2 \gamma}\left|\Gamma_{0}^{\prime}\right|$, then $\left|\Gamma_{0}^{\prime}\right| \geq \frac{2 \gamma}{2 \gamma+1}\left|\Gamma_{0}\right|$. Moreover, the two neighbours of $\Gamma_{0}^{\prime}$ in $\pi_{n_{1}}$ are $\Lambda$ and $\Gamma_{0}^{\prime \prime}$, both of length $\leq \frac{1}{2 \gamma}\left|\Gamma_{0}^{\prime}\right|$. Therefore, by Lemma 3.5(i), if $t_{l}$ is the first knot in $\mathcal{T}$ with $l>n_{1}$ and belonging to $\Lambda \cup \Gamma_{0}^{\prime} \cup \Gamma_{0}^{\prime \prime}$, then $t_{l} \in \Gamma_{0}^{\prime}$. Now $t_{l}$ splits $\Gamma_{0}^{\prime}$ into two parts: $\left(\Gamma_{0}^{\prime}\right)^{\prime}$, adjacent to $\Lambda$, and $\left(\Gamma_{0}^{\prime}\right)^{\prime \prime}$, adjacent to $\Gamma_{0}^{\prime \prime}$. Lemma 3.5(i) also shows that both these intervals are of length $\geq \frac{1}{2 \gamma}\left|\Gamma_{0}^{\prime}\right| \geq \frac{1}{2 \gamma+1}\left|\Gamma_{0}\right|$. In this case we put $\Gamma_{1}=\left(\Gamma_{0}^{\prime}\right)^{\prime}=\left[\tau, t_{l}\right]$ and $L_{1}=\left(\Gamma_{0}^{\prime}\right)^{\prime \prime}=\left[t_{l}, t_{n_{1}}\right]$, and (4.14) is checked as in the previous case.

Now, by an induction argument, for each $i, 0 \leq i \leq k-1$, having an interval $\Gamma_{i}$ adjacent to $\Lambda$ and such that $|\Lambda| \leq\left(\frac{1}{4 \gamma}\right)^{k-i+\varrho}\left|\Gamma_{i}\right|$, we find $n_{i+1}$ and disjoint intervals $\Gamma_{i+1}, L_{i+1} \subset \Gamma_{i}$ such that $\Lambda$ and $\Gamma_{i+1}$ are neighbouring intervals in some partition $\pi_{l}$ with $l \geq n_{i+1}$, and

$$
|\Lambda| \leq\left(\frac{1}{4 \gamma}\right)^{k-(i+1)+\varrho_{\gamma}}\left|\Gamma_{i+1}\right|, \quad \int_{L_{i+1}}\left|a_{n_{i+1}}(\phi) f_{n_{i+1}}(t)\right| d t \sim_{\gamma} 1 .
$$

It follows from the construction that the intervals $L_{1}, \ldots, L_{k}$ are disjoint. Therefore, for $\phi$ given by (4.13) we have

$$
\int_{0}^{1} \sup _{n \geq 0}\left|a_{n}(\phi) f_{n}(t)\right| d t \geq \sum_{i=1}^{k} \int_{L_{i}}\left|a_{n_{i}}(\phi) f_{n_{i}}(t)\right| d t \sim_{\gamma} k .
$$

As this can be done for each $k \geq 1$, this completes the proof if $|\Lambda|>0$. 
The case of $|\Lambda|=0$ requires only minor changes. In this case, for some $n_{\tau}$, the point $\tau$ is a double knot for all partitions $\pi_{n}$ with $n \geq n_{\tau}$. If $\Gamma$ is an interval of some $\pi_{n}$ with $n>n_{\tau}$ and has left endpoint $\tau$, and $\Gamma^{\prime}, \Gamma^{\prime \prime}$ are intervals obtained by the first splitting of $\Gamma$ by a knot $t_{s}$ with $s>n$ and $t_{s}$ not equal to the right endpoint of $\Gamma$, and with $\Gamma^{\prime}$ having left endpoint $\tau$, then by Lemma 3.5(i) we get $\left|\Gamma^{\prime}\right| \sim_{\gamma}|\Gamma|$. In addition, if $\left|\Gamma^{\prime \prime}\right|<\frac{1}{2 \gamma}\left|\Gamma^{\prime}\right|$, then $\left(\Gamma^{\prime}\right)^{\prime},\left(\Gamma^{\prime}\right)^{\prime \prime}$, i.e. the first splitting of $\Gamma^{\prime}$, satisfies $\left|\left(\Gamma^{\prime}\right)^{\prime}\right| \sim_{\gamma}\left|\left(\Gamma^{\prime}\right)^{\prime \prime}\right| \sim_{\gamma}\left|\Gamma^{\prime}\right|$. Therefore, we can find an infinite increasing sequence $\left(n_{i}, i \geq 1\right)$ and associated intervals $\Gamma_{n_{i}}$ such that $\Gamma_{n_{i}}$ is an interval of $\pi_{n_{i}-1}$ with left endpoint $\tau$, $t_{n_{i}} \in \Gamma_{n_{i}}$ and $t_{n_{i}}$ is a simple knot in $\pi_{n_{i}}$, and the splitting $\Gamma_{n_{i}}^{\prime}, \Gamma_{n_{i}}^{\prime \prime}$ of $\Gamma_{n_{i}}$ by $t_{n_{i}}$ satisfies $\left|\Gamma_{n_{i}}^{\prime}\right| \sim_{\gamma}\left|\Gamma_{n_{i}}^{\prime \prime}\right| \sim_{\gamma}\left|\Gamma_{n_{i}}\right|$, where $\Gamma_{n_{i}}^{\prime}$ has left endpoint $\tau$. It follows that $\Gamma_{n_{i+1}} \subset \Gamma_{n_{i}}^{\prime}$ and the intervals $\Gamma_{n_{i}}^{\prime \prime}$ are pairwise disjoint. Observe that $\tau=t_{n_{i}}^{-}$, and by (3.4) in Proposition 3.1,

$$
\lim _{t \rightarrow t_{n_{i}}^{-}+0}\left|f_{n_{i}}(t)\right|,\left|f_{n_{i}}\left(t_{n_{i}}\right)\right|, \lim _{t \rightarrow t_{n_{i}}^{+}-0}\left|f_{n_{i}}(t)\right| \sim_{\gamma}\left|\Gamma_{n_{i}}\right|^{-1 / 2} .
$$

Moreover, as $\tau$ is a double knot in $\pi_{n_{i}-1}$, we have $f_{n_{i}}(t)=0$ for $t \in[0, \tau)$ (cf. Proposition 3.1). Now, let $k \geq 1$, and let

$$
\phi_{k}=\frac{1}{2\left|\Gamma_{n_{k}}^{\prime}\right|}\left(\chi_{\left[\tau-\left|\Gamma_{n_{k}}^{\prime}\right|, \tau\right]}-\chi_{\Gamma_{n_{k}}^{\prime}}\right)
$$

Clearly, $\phi_{k}$ is an atom, and by arguments analogous to the previous case, $\left|a_{n_{i}}\left(\phi_{k}\right)\right| \sim_{\gamma}\left|\Gamma_{n_{i}}\right|^{-1 / 2}$ for $1 \leq i \leq k$. Consequently,

$$
\int_{0}^{1} \sup _{n \geq 0}\left|a_{n}\left(\phi_{k}\right) f_{n}(t)\right| d t \geq \sum_{i=1}^{k} \int_{\Gamma_{n_{i}}^{\prime \prime}}\left|a_{n_{i}}\left(\phi_{k}\right) f_{n_{i}}(t)\right| d t \sim_{\gamma} k .
$$

This completes the proof of Proposition 4.6.

Implication $(\mathrm{C}) \Rightarrow(\mathrm{D})$. We show that this implication holds for partitions satisfying the strong regularity condition for pairs.

Proposition 4.7. Let $\mathcal{T}$ be an admissible sequence of knots with the corresponding general Franklin system $\left\{f_{n}, n \geq 0\right\}$. Let $\gamma \geq 1$ and assume that $\mathcal{T}$ satisfies the strong regularity condition for pairs with parameter $\gamma$. Let $\left(a_{n}, n \geq 0\right)$ be a sequence of coefficients such that $S \in L^{1}[0,1]$. Then there is $f \in H^{1}[0,1]$ such that $a_{n}=\left(f, f_{n}\right)$ for each $n \geq 0$. Moreover, there is a constant $C_{\gamma}$, depending only on $\gamma$, such that for each $f \in H^{1}[0,1]$,

$$
\|f\|_{H^{1}} \leq C_{\gamma}\|S f\|_{1} .
$$

Proof. If $S \in L^{1}[0,1]$, then there is $f \in L^{1}[0,1]$ such that $f=\sum_{n=0}^{\infty} a_{n} f_{n}$, with the series convergent in $L^{1}[0,1]$; this follows by the relative weak compactness in $L^{1}[0,1]$ of a uniformly integrable subset. We need to show that $f \in H^{1}[0,1]$, and for this, we find a suitable atomic decomposition of $f$. 
For convenience, assume that $\|S\|_{1}=1$. Let $E_{0}=B_{0}=[0,1]$, and for $r \geq 1$,

$$
\begin{aligned}
& E_{r}=\left\{t \in[0,1]: S(t)>2^{r}\right\}, \\
& B_{r}=\left\{t \in[0,1]: \mathcal{M}\left(\chi_{E_{r}}, t\right)>\frac{1}{8 \gamma+5}\right\} .
\end{aligned}
$$

Since $\mathcal{M}$ is of weak type $(1,1)$, we have $\left|B_{r}\right| \leq C_{\gamma}\left|E_{r}\right|$. As $\|S\|_{1}=1$, we have $\left|E_{r}\right|,\left|B_{r}\right| \rightarrow 0$ as $r \rightarrow \infty$.

Now,

$$
B_{r}=\bigcup_{\kappa \in \Omega_{r}} \Gamma_{r, \kappa}
$$

where the set $\Omega_{r}$ of indices is at most countable, $\left\{\Gamma_{r, \kappa}: \kappa \in \Omega\right\}$ is a collection of disjoint intervals no two of which have a common endpoint, and the equality in (4.17) is up to a set of zero Lebesgue measure. As $B_{r}$ is an open set, the collection $\left\{\Gamma_{r, \kappa}: \kappa \in \Omega_{r}\right\}$ can be taken as the collection of level sets of positive measure of the continuous nondecreasing function $h_{r}(t)=\left|[0, t] \cap B_{r}^{\mathrm{c}}\right|$. Observe that if $\Gamma_{r+1, \xi}$ appears in the representation (4.17) of $B_{r+1}$, then in the representation (4.17) of $B_{r}$ there is $\Gamma_{r, \kappa}$ such that $\Gamma_{r+1, \xi} \subset \Gamma_{r, \kappa}$.

Consider the following sequence of functions: $g_{0} \equiv \int_{0}^{1} f(t) d t$, and for $r \geq 1$,

$$
g_{r}(t)= \begin{cases}f(t) & \text { for } t \in B_{r}^{\mathrm{c}}, \\ \frac{1}{\left|\Gamma_{r, \kappa}\right|} \int_{\Gamma_{r, \kappa}} f(u) d u & \text { for } t \in \Gamma_{r, \kappa} .\end{cases}
$$

Observe that $f=g_{0}+\sum_{r=0}^{\infty}\left(g_{r+1}-g_{r}\right)$, with the series convergent in $L^{1}$. As $B_{r+1} \subset B_{r}$, it follows from the definition of $g_{r}, g_{r+1}$ that $g_{r+1}-g_{r}=0$ on $B_{r}^{\mathrm{c}}$. In addition, for each $r, \kappa$ we have

$$
\begin{aligned}
\int_{\Gamma_{r, \kappa}} g_{r+1}(t) d t & =\int_{\Gamma_{r, \kappa} \cap B_{r+1}^{\mathrm{c}}} g_{r+1}(t) d t+\int_{\Gamma_{r, \kappa} \cap B_{r+1}} g_{r+1}(t) d t \\
& =\int_{\Gamma_{r, \kappa} \cap B_{r+1}^{\mathrm{c}}} f(t) d t+\sum_{\xi \in \Omega_{r+1}: \Gamma_{r+1, \xi} \subset \Gamma_{r, \kappa}} \int_{\Gamma_{r+1, \xi}} f(t) d t \\
& =\int_{\Gamma_{r, \kappa}} f(t) d t=\int_{\Gamma_{r, \kappa}} g_{r}(t) d t .
\end{aligned}
$$

The main step in the proof is to show that

$$
\left|g_{r}(t)\right| \leq C_{\gamma} 2^{r} \quad \text { almost everywhere on }[0,1] .
$$

Once this inequality is proved, we take $\phi_{0} \equiv 1, \eta_{0}=\int_{0}^{1} f(u) d u$, and

$$
\phi_{r, \kappa}=\frac{\left(g_{r+1}-g_{r}\right) \chi_{\Gamma_{r, \kappa}}}{C_{\gamma} 2^{r}\left|\Gamma_{r, \kappa}\right|}, \quad \eta_{r, \kappa}=C_{\gamma} 2^{r}\left|\Gamma_{r, \kappa}\right| \quad \text { for } r \geq 0, \kappa \in \Omega_{r} .
$$


It then follows that $\phi_{0}, \phi_{r, \kappa}$ are atoms, and

$$
\begin{aligned}
\left|\eta_{0}\right|+\sum_{r=0}^{\infty} \sum_{\kappa \in \Omega_{r}}\left|\eta_{r, \kappa}\right| & \leq\left|\int_{0}^{1} f(t) d t\right|+C_{\gamma} \sum_{r=0}^{\infty} \sum_{\kappa \in \Omega_{r}} 2^{r}\left|\Gamma_{r, \kappa}\right| \\
& \leq\|S\|_{1}+C_{\gamma} \sum_{r=0}^{\infty} 2^{r}\left|B_{r}\right| \\
& \leq\|S\|_{1}+C_{\gamma} \sum_{r=0}^{\infty} 2^{r}\left|E_{r}\right| \leq C_{\gamma}\|S\|_{1}=C_{\gamma} .
\end{aligned}
$$

This implies that $f=\eta_{0} \phi_{0}+\sum_{r=0}^{\infty} \sum_{\kappa \in \Omega_{r}} \eta_{r, \kappa} \phi_{r, \kappa}$ is an atomic decomposition of $f$, and $\|f\|_{H^{1}} \leq C_{\gamma}$.

Thus, it remains to prove (4.19). First, let $t \in B_{r}^{\mathrm{c}}$ and let $t$ be a Lebesgue point of $f$. It is enough to consider $t \notin \mathcal{T}$. Fix $m \geq 0$, and let $V_{m}$ be an interval of linearity of $S_{m}=\sum_{n=0}^{m} a_{n} f_{n}$ containing $t$. Then $V_{m} \not \subset B_{r}$, and consequently $\left|V_{m} \cap E_{r}^{\mathrm{c}}\right| \geq \frac{1}{2}\left|V_{m}\right|$. Since $\left|S_{m}\right| \leq 2^{r}$ on $E_{r}^{\mathrm{c}}$, it follows by Fact 4.1 that $\left|S_{m}\right| \leq C 2^{r}$ on $V_{m}$, and in particular $\left|S_{m}(t)\right| \leq C 2^{r}$. Since $S_{m}(t) \rightarrow f(t)$ as $m \rightarrow \infty$ (cf. Proposition 3.6(ii)), this implies that $\left|g_{r}(t)\right|=|f(t)| \leq S(t) \leq C 2^{r}$.

Now, fix $\kappa \in \Omega_{r}$, and consider $g_{r}$ on $\Gamma=\Gamma_{r, \kappa}$. For further convenience, write $\Gamma=[\alpha, \beta]$. Let $N(\Gamma)$ be the collection of indices $n \geq 0$ satisfying one

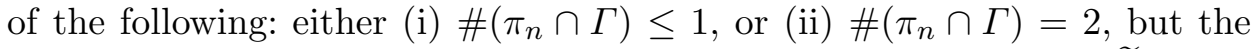
interval $V \subset \Gamma$ of the partition $\pi_{n}$ is "short" in the sense that if $\widetilde{V}$ is the "parent interval" for $V$, i.e. $V$ is one of the two intervals obtained from the first splitting of $\widetilde{V}$ by a knot from $\mathcal{T}$, then $|V| \leq|\widetilde{V}| / 2$. (Recall that to find $\#\left(\pi_{n} \cap \Gamma\right)$, we count knots of $\pi_{n}$ with their multiplicities in $\pi_{n}$.) In case (ii), the situation that $V$ is a degenerate interval, i.e. $|V|=0$, is also allowed.

Note that if $n \in N(\Gamma)$ and $n^{\prime}<n$ then $n^{\prime} \in N(\Gamma)$. Let $n_{\Gamma}=\max N(\Gamma)+1$. It follows by the definition of $n_{\Gamma}$ that $t_{n_{\Gamma}} \in \Gamma$. Let $U_{0}, W_{0}$ be the intervals of the partition $\pi_{n_{\Gamma}}$, containing $\alpha$ and $\beta$, respectively. In case $\alpha$ (respectively $\beta$ ) is a knot of $\pi_{n_{\Gamma}}$, then $U_{0}$ (respectively $W_{0}$ ) is a nondegenerate interval of $\pi_{n_{\Gamma}}$ with right endpoint $\alpha$ (respectively, left endpoint $\beta$ ). It should be clear that the interiors of $U_{0}, W_{0}$ are disjoint.

Let $S_{\Gamma}=\sum_{n=0}^{n_{\Gamma}} a_{n} f_{n}$. Note that $\left|U_{0} \cap B_{r}\right|<\left|U_{0}\right|$; otherwise, $U_{0} \cup \Gamma$ would replace $\Gamma$ in the representation (4.17). If $\max _{U_{0}}\left|S_{\Gamma}\right|>M_{1 / 2} 2^{r}$, then by Fact $4.1,\left|S_{\Gamma}\right|>2^{r}$ on a subset of $U_{0}$ of measure $\left|U_{0}\right| / 2$, and consequently $\left|U_{0} \cap E_{r}\right| \geq\left|U_{0}\right| / 2$. But then $U_{0} \subset B_{r}$, contrary to $\left|U_{0} \cap B_{r}\right|<\left|U_{0}\right|$. Thus $\max _{U_{0}}\left|S_{\Gamma}\right| \leq M_{1 / 2} 2^{r}$. By an analogous argument we have $\max _{W_{0}}\left|S_{\Gamma}\right| \leq$ $M_{1 / 2} 2^{r}$.

The next step is to prove that $\left|U_{0}\right|,\left|W_{0}\right| \leq 2 \gamma|\Gamma|$ and $\left|S_{\Gamma}\right| \leq M_{1 / 2} 2^{r}$ on $\Gamma$. Consider two cases. First suppose that all $n \in N(\Gamma)$ satisfy (i). Then $\#\left(\pi_{n_{\Gamma}} \cap \Gamma\right)=2$, but the interval $V$ of $\pi_{n_{\Gamma}}$ included in $\Gamma$ is "long", i.e. 
$|V|>|\widetilde{V}| / 2$, where $\widetilde{V}$ is the "parent interval" for $V$. Since $t_{n_{\Gamma}}$ is one of the endpoints of $V$, either $\widetilde{V}=V \cup U_{0}$ or $\widetilde{V}=V \cup W_{0}$. Suppose that $\widetilde{V}=V \cup U_{0}$. Then $\left|U_{0}\right| \leq|V| \leq|\Gamma|$. Moreover, $U_{0}, V$ and $V, W_{0}$ are neighbouring pairs in $\pi_{n_{\Gamma}}$, so by the strong regularity for pairs,

$$
\left|W_{0}\right| \leq\left|W_{0}\right|+|V| \leq \gamma\left(|V|+\left|U_{0}\right|\right) \leq 2 \gamma|V| \leq 2 \gamma|\Gamma| .
$$

The case $\widetilde{V}=V \cup W_{0}$ is analogous. Note that in both cases we have $\left|U_{0}\right|+$ $\left|W_{0}\right| \leq(2 \gamma+1)|V|$. On the other hand, $\Gamma \subset U_{0} \cup V \cup W_{0}$, which implies $|\Gamma| \leq$ $(2 \gamma+2)|V|$. If we had $\max _{V}\left|S_{\Gamma}\right|>M_{1 / 2} 2^{r}$, then by Fact $4.1,\left|S_{\Gamma}\right|>2^{r}$ on a subset of $V$ of measure $|V| / 2$, so $\left|V \cap E_{r}\right| \geq|V| / 2$. Let $\Delta=U_{0} \cup V \cup W_{0}$. Note that $\Delta$ is an interval properly containing $\Gamma$, and $|V| \leq|\Delta| \leq(2 \gamma+2)|V|$. Thus, we would have

$$
\left|\Delta \cap E_{r}\right| \geq\left|V \cap E_{r}\right| \geq \frac{|V|}{2} \geq \frac{|\Delta|}{4 \gamma+4} .
$$

This would imply $\Delta \subset B_{r}$, so $\Gamma$ could not be one of intervals in the representation (4.19), which contradicts the definition of $\Gamma$. Thus, $\left|S_{\Gamma}\right| \leq M_{1 / 2} 2^{r}$ on $V$. Putting together this inequality, the previous estimates for $\left|S_{\Gamma}\right|$ on $U_{0}$ and $W_{0}$ and the fact that $\Gamma \subset U_{0} \cup V \cup W_{0}$, we infer that $\left|S_{\Gamma}\right| \leq M_{1 / 2} 2^{r}$ on $\Gamma$.

Now, let us consider the case when some $n \in N(\Gamma)$ satisfy (ii). Then $\#\left(\pi_{n_{\Gamma}} \cap \Gamma\right)=3$ and $\Gamma$ contains two neighbouring intervals of $\pi_{n_{\Gamma}}$, say $V^{\prime}, V^{\prime \prime}$ (one of them may be degenerate); for convenience, we assume that $V^{\prime}$ is to the left of $V^{\prime \prime}$ and $\left|V^{\prime}\right| \geq\left|V^{\prime \prime}\right|$. Then $\left(U_{0}, V^{\prime}\right),\left(V^{\prime}, V^{\prime \prime}\right),\left(V^{\prime \prime}, W_{0}\right)$ are three consecutive pairs of intervals of $\pi_{n_{\Gamma}}$. Then by strong regularity for pairs,

$$
\left|U_{0}\right| \leq\left|U_{0}\right|+\left|V^{\prime}\right| \leq \gamma\left(\left|V^{\prime}\right|+\left|V^{\prime \prime}\right|\right) \leq \gamma|\Gamma|,
$$

and by an analogous argument $\left|W_{0}\right| \leq \gamma|\Gamma|$. Further, setting $\Delta=U_{0} \cup V^{\prime} \cup$ $V^{\prime \prime} \cup W_{0}$, we find that

$$
|\Delta| \leq\left|U_{0}\right|+\left|V^{\prime}\right|+\left|V^{\prime \prime}\right|+\left|W_{0}\right| \leq(2 \gamma+1)\left(\left|V^{\prime}\right|+\left|V^{\prime \prime}\right|\right) \leq(4 \gamma+2)\left|V^{\prime}\right| .
$$

If we had $\max _{V^{\prime}}\left|S_{\Gamma}\right|>M_{1 / 2} 2^{r}$, then, again by Fact 4.1 , we would get $\left|V^{\prime} \cap E_{r}\right| \geq\left|V^{\prime}\right| / 2$. Consequently, $\left|\Delta \cap E_{r}\right| \geq|\Delta| /(8 \gamma+4)$ and $\Delta \subset B_{r}$, which contradicts the definition of $\Gamma$. Therefore, $\max _{V^{\prime}}\left|S_{\Gamma}\right| \leq M_{1 / 2} 2^{r}$. It remains to consider $S_{\Gamma}$ on $V^{\prime \prime}$, and we need to do this only for $\left|V^{\prime \prime}\right|>0$. But then, since there are only three knots of $\pi_{n_{\Gamma}}$ in $\Gamma$, and one of them is the left endpoint of $V^{\prime}$, both endpoints of $V^{\prime \prime}$ are simple knots in $\pi_{n_{\Gamma}}$. This means that $S_{\Gamma}$ is continuous at the endpoints of $V^{\prime \prime}$. Suppose now that $\max _{V^{\prime \prime}}\left|S_{\Gamma}\right|>M_{1 / 2} 2^{r}$. Since $S_{\Gamma}$ is linear on $V^{\prime \prime},\left|S_{\Gamma}\right|$ takes its maximum at one of the endpoints of $V^{\prime \prime}$. But the left endpoint of $V^{\prime \prime}$ is the right endpoint of $V^{\prime}$, and the right endpoint of $V^{\prime \prime}$ is the left endpoint of $W_{0}$. This implies that if $\max _{V^{\prime \prime}}\left|S_{\Gamma}\right|>M_{1 / 2} 2^{r}$, then either $\max _{V^{\prime}}\left|S_{\Gamma}\right|>M_{1 / 2} 2^{r}$ 
or $\max _{W_{0}}\left|S_{\Gamma}\right|>M_{1 / 2} 2^{r}$; but we have already proved that none of these is possible. Therefore, $\max _{V^{\prime \prime}}\left|S_{\Gamma}\right| \leq M_{1 / 2} 2^{r}$.

Summarizing the above considerations, we have shown

$$
\left|U_{0}\right|,\left|W_{0}\right| \leq 2 \gamma|\Gamma|, \quad\left|S_{\Gamma}\right| \leq M_{1 / 2} 2^{r} \quad \text { on } U_{0}, W_{0} \text { and } \Gamma .
$$

In particular, this implies that

$$
\int_{\Gamma}\left|S_{\Gamma}(t)\right| d t \leq M_{1 / 2} 2^{r}|\Gamma|
$$

Next, we need to estimate $\left|\int_{\Gamma} \sum_{n=n_{\Gamma}+1}^{\infty} a_{n} f_{n}(t) d t\right|$. For this, we define inductively two sequences $\left\{\left(u_{i}, U_{i}\right), i \geq 0\right\}$ and $\left\{\left(w_{i}, W_{i}\right), i \geq 0\right\}$ of indices and intervals: put $u_{0}=n_{\Gamma}, w_{0}=n_{\Gamma}$, and $U_{0}, W_{0}$ as above. Having defined $\left(u_{i}, U_{i}\right)$ for $0 \leq i \leq s$, we proceed as follows: if $\alpha$ is a double knot in $\pi_{u_{s}}$, then the procedure terminates. Otherwise, let $u_{s+1}$ be the first $n>u_{s}$ such that $t_{n} \in U_{s}$. If $\alpha$ is not a knot of $\pi_{u_{s+1}}$, then $U_{s+1}$ is defined to be the unique interval of this partition containing $\alpha$; if $\alpha$ is a knot of $\pi_{u_{s+1}}$ (note that it is not a double knot of $\pi_{u_{s}}$ ), then $U_{s+1}$ is a nondegenerate interval of $\pi_{u_{s+1}}$ with right endpoint $\alpha$.

The sequence $\left(w_{i}, W_{i}\right)$ is defined analogously, by using the point $\beta$ and choosing nondegenerate intervals with left endpoint $\beta$.

It follows from the construction that $U_{i+1} \subset U_{i}$ and $W_{i+1} \subset W_{i}$. Moreover, by arguments analogous to those used for $U_{0}, W_{0}$ we find that $U_{i} \not \subset B_{r}$ and $W_{i} \not \subset B_{r}$. In addition, the interiors of $U_{i}$ and $W_{j}$ are disjoint for any $i, j$.

Assume now that $\alpha$ and $\beta$ are not double knots in $\mathcal{T}$. For a pair of indices $i, j$, let $\Phi_{i, j}$ be the following piecewise linear function: $\Phi_{i, j}$ is equal to 0 to the left of the left endpoint of $U_{i}$ and to the right of the right endpoint of $W_{j}$, it is 1 between the right endpoint of $U_{i}$ and the left endpoint of $W_{j}$, and it is linear on $U_{i}$ and $W_{j}$. Then $\Phi_{i, j}$ is piecewise linear with knots $\pi_{u_{i} \vee w_{j}}$. Note that $x_{i}=\Phi_{i, j} \cdot \chi_{U_{i}}$ does not depend on $W_{j}$ and $y_{j}=\Phi_{i, j} \cdot \chi_{W_{j}}$ does not depend on $U_{i}$.

For $i, j \geq 0$, consider two splittings of the set of indices $\left\{n: n>n_{\Gamma}\right\}$ :

$$
L(i)=\left\{n: u_{i}<n \leq u_{i+1}\right\}, \quad R(j)=\left\{n: w_{j}<n \leq w_{j+1}\right\} .
$$

For $n \in L(i) \cap R(j)$ we have $\left(f_{n}, \Phi_{i, j}\right)=0$, and consequently

$$
\int_{\Gamma} f_{n}(t) d t=\int_{\Gamma} f_{n}(t) d t-\int_{0}^{1} f_{n}(t) \Phi_{i, j}(t) d t=A_{i}\left(f_{n}\right)+B_{j}\left(f_{n}\right),
$$

where

$$
\begin{aligned}
& A_{i}\left(f_{n}\right)=\int_{\Gamma \cap U_{i}} f_{n}-\int_{U_{i}} f_{n}(t) x_{i}(t) d t \\
& B_{j}\left(f_{n}\right)=\int_{\Gamma \cap W_{j}} f_{n}-\int_{W_{j}} f_{n}(t) y_{j}(t) d t .
\end{aligned}
$$


This implies

$$
\begin{aligned}
& \left|\int_{\Gamma} \sum_{n=n_{\Gamma}+1}^{\infty} a_{n} f_{n}(t) d t\right|=\left|\sum_{i, j=0}^{\infty} \sum_{n \in L(i) \cap R(j)} a_{n}\left(A_{i}\left(f_{n}\right)+B_{j}\left(f_{n}\right)\right)\right| \\
& \quad \leq 2 \sum_{i=0}^{\infty} \int_{U_{i}}\left|\sum_{n \in L(i)} a_{n} f_{n}(t)\right| d t+2 \sum_{j=0}^{\infty} \int_{W_{j}}\left|\sum_{n \in R(j)} a_{n} f_{n}(t)\right| d t .
\end{aligned}
$$

Consider the first sum on the right hand side. Observe that $\sum_{n \in L(i)} a_{n} f_{n}(t)$ is linear on $U_{i}$. Therefore, if $\max _{U_{i}}\left|\sum_{n \in L(i)} a_{n} f_{n}(t)\right|>M_{1 / 2} 2^{r+1}$, then by Fact $4.1,\left|\sum_{n \in L(i)} a_{n} f_{n}\right|>2^{r+1}$ on a set $U_{i}^{*} \subset U_{i}$ with $\left|U_{i}^{*}\right|=\left|U_{i}\right| / 2$. But this implies that $\max \left(\left|\sum_{n=0}^{u_{i}} a_{n} f_{n}\right|,\left|\sum_{n=0}^{u_{i+1}} a_{n} f_{n}\right|\right)>2^{r}$ on $U_{i}^{*}$. Consequently, $\left|E_{r} \cap U_{i}\right| \geq\left|U_{i}^{*}\right| \geq\left|U_{i}\right| / 2$ and $U_{i} \subset B_{r}$, which is impossible. Thus, $\max _{U_{i}}\left|\sum_{n \in L(i)} a_{n} f_{n}(t)\right| \leq M_{1 / 2} 2^{r+1}$, which gives

$$
\int_{U_{i}}\left|\sum_{n \in L(i)} a_{n} f_{n}(t)\right| d t \leq M_{1 / 2} 2^{r+1}\left|U_{i}\right| .
$$

Combining Lemma 3.5(iii), the inclusions $U_{i+1} \subset U_{i}$ and the inequality $\left|U_{0}\right| \leq 2 \gamma|\Gamma|$ we see that $\sum_{i=0}^{\infty}\left|U_{i}\right| \leq C_{\gamma}|\Gamma|$. Thus, we get

$$
\sum_{i=0}^{\infty} \int_{U_{i}}\left|\sum_{n \in L(i)} a_{n} f_{n}(t)\right| d t \leq C_{\gamma} 2^{r}|\Gamma| .
$$

The second sum on the right hand side of (4.22) is estimated analogously, giving

$$
\sum_{j=0}^{\infty} \int_{W_{j}}\left|\sum_{n \in R(j)} a_{n} f_{n}(t)\right| d t \leq C_{\gamma} 2^{r}|\Gamma| .
$$

Combining these estimates with (4.22) and (4.20) we find

$$
\left|\int_{\Gamma} f(t) d t\right|=\left|\int_{\Gamma} \sum_{n=0}^{\infty} a_{n} f_{n}(t) d t\right| \leq C_{\gamma} 2^{r}|\Gamma| .
$$

This implies inequality (4.19) on $\Gamma$.

If $\alpha$ is a double knot in $\mathcal{T}$, then let $\eta$ be such that $u_{\eta}$ is the last $u_{i}$ chosen before the end of the procedure of choosing $\left(u_{i}, U_{i}\right)$ 's. For $i<\eta, \Phi_{i, j}$ and $L_{i}$ are defined as previously. In addition, we put $L(\eta)=\left\{n: n>u_{\eta}\right\}$ and take modified functions $\Phi_{\eta *, j}: \Phi_{\eta *, j}$ is equal to 0 on $[0, \alpha]$ and to the right of the right endpoint of $W_{j}$, it is 1 between $\alpha$ and the left endpoint of $W_{j}$, and linear on $W_{j}$. Note that $\Phi_{\eta *, j}$ is a piecewise linear function with knots $\pi_{u_{\eta} \vee w_{j}}$. Now, for $n \in L(\eta)$ we write formula (4.21) with $\Phi_{\eta *, j}$ for suitable $j$. Note that then the support of $f_{n}$ is included either in $[0, \alpha]$ or in $[\alpha, 1]$ (cf. Proposition 3.1), and consequently the "left" term $A_{\eta *}\left(f_{n}\right)$ is zero. 
If $\beta$ is a double knot in $\mathcal{T}$, then take $\zeta$ to be such that $w_{\zeta}$ is the last $w_{j}$ chosen. As above, take the modified functions $\Phi_{i, \zeta *}$ having $\beta$ as a double knot, and for $n>w_{\zeta}$, write formula (4.21) with $\Phi_{i, \zeta *}$. Then the "right" term $B_{\zeta^{*}}\left(f_{n}\right)$ is zero.

If both $\alpha$ and $\beta$ are double knots in $\mathcal{T}$, then for $n>u_{\eta} \vee w_{\zeta}$ the support of $f_{n}$ is included either in $\Gamma,[0, \alpha]$, or $[\beta, 1]$, according as $t_{n} \in \Gamma, t_{n}<\alpha$ or $t_{n}>\beta$. In any of these cases we have $\int_{\Gamma} f_{n}(t) d t=0$.

This completes the proof of Proposition 4.7.

\section{PROOFS OF THE MAIN RESULTS}

5.1. Proof of Theorem 2.1. Recall that $\mathcal{T}$ is an admissible sequence of knots in $[0,1]$, with the corresponding Franklin system $\left\{f_{n}, n \geq 0\right\}$. Note that the continuous functions are dense in $H^{1}[0,1]$, and for $f \in C[0,1]$, $\left\|f-Q_{\pi_{n}} f\right\|_{\infty} \rightarrow 0$ as $n \rightarrow \infty$. As $\left\|f-Q_{\pi_{n}} f\right\|_{H^{1}} \leq\left\|f-Q_{\pi_{n}} f\right\|_{\infty}$, this implies that the collection $\left\{f_{n}, n \geq 0\right\}$ is linearly dense in $H^{1}[0,1]$. Clearly, it is also minimal. Therefore, $\left\{f_{n}, n \geq 0\right\}$ is a basis in $H^{1}[0,1]$ if and only if the corresponding sequence of partial sum operators is bounded in $H^{1}[0,1]$, that is, iff there is a constant $C_{\mathcal{T}}$ such that

$$
\left\|Q_{\pi_{n}}\right\|_{H^{1}}=\left\|Q_{\pi_{n}}: H^{1}[0,1] \rightarrow H^{1}[0,1]\right\| \leq C_{\mathcal{T}} \quad \text { for all } n \geq 0 .
$$

For the proof of the necessity part, let us recall Lemma 5.2 of [9] (version for $p=1)$ :

Lemma 5.1. Let $\varepsilon>0$ and let $\pi=\left\{\tau_{i}, 0 \leq i \leq m\right\}$ be a partition of $[0,1]$ such that there exist three consecutive intervals $\Lambda_{k-1}, \Lambda_{k}, \Lambda_{k+1}$, where $\Lambda_{l}=\left[\tau_{l-1}, \tau_{l}\right]$, with the following property: either

$$
\left|\Lambda_{k+1}\right| \leq \varepsilon\left|\Lambda_{k-1}\right| \quad \text { and }\left|\Lambda_{k}\right| \leq \varepsilon\left|\Lambda_{k-1}\right|,
$$

or

$$
\left|\Lambda_{k-1}\right| \leq \varepsilon\left|\Lambda_{k+1}\right| \quad \text { and } \quad\left|\Lambda_{k}\right| \leq \varepsilon\left|\Lambda_{k+1}\right| .
$$

Let $Q_{\pi}$ be the orthogonal projection onto $S_{\pi}$. Then there are $\varepsilon_{0}>0$ and $C>0$ such that for all partitions $\pi$ satisfying the above condition with $0<\varepsilon \leq \varepsilon_{0}$,

$$
\left\|Q_{\pi}\right\|_{H^{1}} \geq C \log (1 / \varepsilon) .
$$

Lemma 5.2 of [9] has been proved for partitions with simple knots. Let us discuss briefly the changes needed in the proof when double knots are allowed. The proof in [9] proceeds by indicating an atom $\phi$ for which $\left\|Q_{\pi} \phi\right\|_{H^{1}} \geq C \log (1 / \varepsilon)$. Let us consider the case when the first set of inequalities in Lemma 5.1 is satisfied. If $\left|\Lambda_{k}\right|>0$, then both the choice of the atom

$$
\phi=\frac{\chi_{\Lambda_{k}}-\chi_{\left[\tau_{k-1}-\left|\Lambda_{k}\right|, \tau_{k-1}\right]}}{\left|\Lambda_{k}\right|}
$$


and further considerations are as in the proof of Lemma 5.2 of [9], even if $\left|\Lambda_{k+1}\right|=0$, or some knot of $\pi$ other than $\tau_{k}$ is double. If $\left|\Lambda_{k}\right|=0$, i.e. $\tau_{k}=\tau_{k-1}$, then $\left|\Lambda_{k+1}\right|>0$, and it is enough to consider

$$
\phi(t)=2 \frac{\tau_{k+1}-t}{\left|\Lambda_{k+1}\right|^{2}} \chi_{\Lambda_{k+1}}(t)-\frac{1}{\left|\Lambda_{k+1}\right|} \chi_{\left[\tau_{k}-\left|\Lambda_{k+1}\right|, \tau_{k}\right]}(t) .
$$

Since $\tau_{k}=\tau_{k-1}$ is a double knot of $\pi$, for this $\phi$ we have

$$
Q_{\pi} \phi(t)=2 \frac{\tau_{k+1}-t}{\left|\Lambda_{k+1}\right|^{2}} \chi_{\Lambda_{k+1}}(t) \quad \text { for } t>\tau_{k},
$$

and by arguments analogous to those from [9], $\left|Q_{\pi} \phi(t)\right| \leq 15 /\left|\Lambda_{k-1}\right|$ for $t \leq \tau_{k}$. When we know this, considerations analogous to those in [9] (with $\tau_{k}$ replacing $\left.y_{k}\right)$ give the required lower bound for $\left\|Q_{\pi} \phi\right\|_{H^{1}}$.

Now, if $\mathcal{T}$ does not satisfy the strong regularity condition for pairs with any $\gamma>0$, then for each $\varepsilon$ we can find $n_{\varepsilon}$ such that $\pi_{n_{\varepsilon}}$ satisfies the conditions of Lemma 5.1. Consequently, $\left\|Q_{\pi_{n_{\varepsilon}}}\right\|_{H^{1}} \geq C \log (1 / \varepsilon)$, and condition (5.1) is not satisfied.

To complete the proof of Theorem 2.1, we need to show that strong regularity for pairs implies (5.1). This is the content of

Lemma 5.2. Let $\mathcal{T}=\left(t_{i}, i \geq 0\right)$ be a sequence of knots in $[0,1]$ satisfying the strong regularity condition for pairs with parameter $\gamma$. Let $\mathcal{T}_{n}=\left(t_{i}, 0 \leq\right.$ $i \leq n)$, let $\pi_{n}$ be the partition of $[0,1]$ obtained by nonincreasing rearrangement of $\mathcal{T}_{n}$, and let $Q_{\pi_{n}}$ be the orthogonal projection on $S\left(\pi_{n}\right)$. Then there is a constant $C_{\gamma}$ such that for every $n \geq 1$ and every $f \in H^{1}[0,1]$,

$$
\left\|Q_{\pi_{n}} f\right\|_{H^{1}} \leq C_{\gamma}\|f\|_{H^{1}} .
$$

Proof. Write $\pi_{n}=\left(t_{n, i}, 0 \leq i \leq n\right), t_{n, i} \leq t_{n, i+1}$.

First, let $\phi$ be an atom. To simplify the notation, let $\eta=Q_{\pi_{n}} \phi$. We are going to construct a suitable atomic decomposition for $\eta$.

If $\phi \equiv 1$, then $\eta \equiv 1$, and $\eta$ is an atom.

Now, let $\phi$ be an atom such that $\int_{0}^{1} \phi(t) d t=0, \operatorname{supp} \phi \subset \Gamma=[\alpha, \beta]$, and $|\phi| \leq 1 /|\Gamma|$. Moreover, let $\Delta=\left[t_{n, k}, t_{n, l}\right]$ be the minimal interval with endpoints in $\pi_{n}$ containing $\Gamma$. That is, $\Gamma \subset \Delta$, and there are no knots of $\pi_{n}$ in $\left(t_{n, k}, \alpha\right],\left[\beta, t_{n, l}\right)$.

For $0 \leq i \leq n$, let $N_{i}^{-}=N_{\pi_{n}, i} \cdot \chi_{\left(t_{n, i-1}, t_{n, i}\right]}, N_{i}^{+}=N_{\pi_{n}, i} \cdot \chi_{\left(t_{n, i}, t_{n, i+1}\right]}$, with the understanding that $N_{0}^{-} \equiv 0$ and $N_{n}^{+} \equiv 0$; note that if $t_{n, i-1}=t_{n, i}$, then $N_{i-1}^{-}=N_{\pi_{n}, i-1}, N_{i-1}^{+} \equiv 0$ and $N_{i}^{-} \equiv 0, N_{i}^{+}=N_{\pi_{n}, i}$.

As $\eta \in S\left(\pi_{n}\right)$, there are coefficients $\left(a_{i}, 0 \leq i \leq n\right)$ such that $\eta=$ $\sum_{i=0}^{n} a_{i} N_{\pi_{n}, i}$. Now, introduce the functions

$$
\left\{\begin{array}{l}
\psi_{0}=\frac{2}{3} a_{0} N_{\pi_{n}, 0}+\frac{1}{3} a_{1} N_{1}^{-}, \quad \psi_{n}=\frac{1}{3} a_{n-1} N_{n-1}^{+}+\frac{2}{3} a_{n} N_{\pi_{n}, n} \\
\psi_{i}=\frac{1}{3} a_{i-1} N_{i-1}^{+}+\frac{2}{3} a_{i} N_{\pi_{n}, i}+\frac{1}{3} a_{i+1} N_{i+1}^{-} \quad \text { for } \quad 1 \leq i \leq n-1 .
\end{array}\right.
$$

They will be used to get the required atomic decomposition for $\eta$. 
Since $N_{\pi_{n}, i}=N_{i}^{-}+N_{i}^{+}$, we have

$$
\eta=\sum_{i=0}^{n} \psi_{i}
$$

Set $L_{i}=\operatorname{supp} N_{\pi_{n}, i}$; recall that $\operatorname{supp} N_{\pi_{n}, i}=\left[t_{n, i-1}, t_{n, i+1}\right]$ for $1 \leq i \leq n-1$, $\operatorname{supp} N_{\pi_{n}, 0}=\left[t_{n, 0}, t_{n, 1}\right]$ and $\operatorname{supp} N_{\pi_{n}, n}=\left[t_{n, n-1}, t_{n, n}\right]$. Note that

$$
\operatorname{supp} \psi_{i} \subset L_{i}, \quad 0 \leq i \leq n \text {. }
$$

Moreover, we have

$$
\left\{\begin{array}{l}
\left(\eta, N_{\pi_{n}, 0}\right)=\frac{\lambda_{n, 1}}{3} a_{0}+\frac{\lambda_{n, 1}}{6} a_{1}=\int_{0}^{1} \psi_{0}(t) d t, \\
\left(\eta, N_{\pi_{n}, i}\right)=\frac{\lambda_{n, i}}{6} a_{i-1}+\frac{\lambda_{n, i}+\lambda_{n, i+1}}{3} a_{i}+\frac{\lambda_{n, i+1}}{6} a_{i+1}=\int_{0}^{1} \psi_{i}(t) d t \\
\quad \text { for } 1 \leq i \leq n-1, \\
\left(\eta, N_{\pi_{n}, n}\right)=\frac{\lambda_{n, n}}{6} a_{n-1}+\frac{\lambda_{n, n}}{3} a_{n}=\int_{0}^{1} \psi_{n}(t) d t .
\end{array}\right.
$$

Note that $\left(\eta, N_{\pi_{n}, i}\right)=\left(\phi, N_{\pi_{n}, i}\right)$. Now, we split the system (5.5) into three parts: (I) equations with $0 \leq i \leq k-1$, (II) equations with $k \leq i \leq l$, (III) equations with $l+1 \leq i \leq n$.

First consider the subsystem (I); note that this case appears only when $k>0$. For $0 \leq i \leq k-1$, the supports of $\phi$ and $N_{\pi_{n}, i}$ are disjoint, so $\left(\phi, N_{\pi_{n}, i}\right)=0$. In particular, this implies that

$$
\int_{0}^{1} \psi_{i}(t) d t=0 \quad \text { for } i \leq k-1
$$

By arguments analogous to those used e.g. in the proof of Lemma 5.2 of [9] we get, for $0 \leq i \leq k-1$,

$$
a_{i} a_{i+1} \leq 0, \quad\left|a_{i}\right| \leq \frac{1}{2}\left|a_{i+1}\right|, \quad\left|a_{i}\right| \leq \frac{2}{3} \frac{\lambda_{n, i+1}}{\lambda_{n, i}+\lambda_{n, i+1}}\left|a_{i+1}\right| .
$$

Since $\|\phi\|_{1} \leq 1$ and $\left\|Q_{\pi_{n}}\right\|_{1} \leq 3$ ( see Proposition 3.6(i)), we have $\|\eta\|_{1} \leq 3$. But

$$
\|\eta\|_{1} \sim \sum_{i=0}^{n}\left|a_{i}\right|\left(\lambda_{n, i}+\lambda_{n, i+1}\right) \geq\left|a_{k}\right|\left(\lambda_{n, k}+\lambda_{n, k+1}\right) .
$$

From the last two inequalities we get, for $i \leq k-1$, 


$$
\begin{aligned}
\left|a_{i}\right| & \leq\left(\frac{2}{3}\right)^{k-i} \prod_{j=i}^{k-1} \frac{\lambda_{n, j+1}}{\lambda_{n, j}+\lambda_{n, j+1}}\left|a_{k}\right| \\
& =\left(\frac{2}{3}\right)^{k-i} \frac{\lambda_{n, k}\left|a_{k}\right|}{\lambda_{n, i}+\lambda_{n, i+1}} \prod_{j=i+1}^{k-1} \frac{\lambda_{n, j}}{\lambda_{n, j}+\lambda_{n, j+1}} \leq C\left(\frac{2}{3}\right)^{k-i} \frac{1}{\lambda_{n, i}+\lambda_{n, i+1}} .
\end{aligned}
$$

As $\left\|\psi_{i}\right\|_{\infty} \leq \max \left(\left|a_{i-1}\right|,\left|a_{i}\right|,\left|a_{i+1}\right|\right)$, the above estimates (together with (5.7) in case $i=k-1$ ), strong regularity for pairs and (5.4) imply that

$$
\left|\left(\frac{3}{2}\right)^{k-i} \psi_{i}(t)\right| \leq \frac{C_{\gamma}}{\left|L_{i}\right|} \quad \text { for } t \in L_{i}, i \leq k-1 .
$$

Now, put

$$
\widetilde{\psi}_{i}=\frac{1}{C_{\gamma}}\left(\frac{3}{2}\right)^{k-i} \psi_{i}, \quad b_{i}=C_{\gamma}\left(\frac{2}{3}\right)^{k-i} \quad \text { for } i \leq k-1 .
$$

It follows from (5.6) and (5.8) that $\widetilde{\psi}_{i}, 0 \leq i \leq k-1$, are atoms.

By analogous considerations for the subsystem (III), we get

$$
\int_{0}^{1} \psi_{i}(t) d t=0, \quad\left|\left(\frac{3}{2}\right)^{i-l} \psi_{i}(t)\right| \leq \frac{C_{\gamma}}{\left|L_{i}\right|} \quad \text { for } t \in L_{i}, i \geq l+1 .
$$

Put

$$
\widetilde{\psi}_{i}=\frac{1}{C_{\gamma}}\left(\frac{3}{2}\right)^{i-l} \psi_{i}, \quad b_{i}=C_{\gamma}\left(\frac{2}{3}\right)^{i-l} \quad \text { for } i \geq l+1 .
$$

It follows that $\widetilde{\psi}_{i}, l+1 \leq i \leq n$, are atoms.

It remains to consider the part corresponding to (II). To this end, put $\widetilde{\Delta}=\left[t_{n, k-1}, t_{n, l+1}\right]$ and

$$
\psi^{*}=\psi_{k}+\cdots+\psi_{l}
$$

It follows by the partition of unity property for $N_{\pi_{n}, i}$ 's and $\left(\phi, N_{\pi_{n}, i}\right)=0$ for $i \leq k-1$ and $i \geq l+1$ that

$$
0=\int_{0}^{1} \phi(t) d t=\int_{0}^{1} \phi(t) \sum_{i=0}^{n} N_{\pi_{n}, i}(t) d t=\sum_{i=0}^{n}\left(\phi, N_{\pi_{n}, i}\right)=\sum_{i=k}^{l}\left(\phi, N_{\pi_{n}, i}\right) .
$$

Using this equality, (5.4) and (5.5) we obtain

$$
\operatorname{supp} \psi^{*} \subset \widetilde{\Delta}, \quad \int_{0}^{1} \psi^{*}(t) d t=\sum_{i=k}^{l}\left(\phi, N_{\pi_{n}, i}\right)=0 .
$$

It remains to show that $\left\|\psi^{*}\right\|_{\infty} \leq C_{\gamma} /|\widetilde{\Delta}|$. For this, consider two cases.

(II-a) $l>k+3$. In this case, $\Gamma$ contains the intervals $\left[t_{n, k+1}, t_{n, k+3}\right]$ and $\left[t_{n, l-3}, t_{n, l-1}\right]$. By strong regularity for pairs,

$$
\left|\left[t_{n, k-1}, t_{n, k+1}\right]\right| \sim_{\gamma}\left|\left[t_{n, k+1}, t_{n, k+3}\right]\right|, \quad\left|\left[t_{n, l-3}, t_{n, l-1}\right]\right| \sim_{\gamma}\left|\left[t_{n, l-1}, t_{n, l+1}\right]\right| .
$$


Since $|\widetilde{\Delta}| \sim|\Gamma|+\left|\left[t_{n, k-1}, t_{n, k+1}\right]\right|+\left|\left[t_{n, l-1}, t_{n, l+1}\right]\right|$, we find that

$$
|\widetilde{\Delta}| \sim_{\gamma}|\Gamma| .
$$

Further, since $\|\phi\|_{\infty} \leq 1 /|\Gamma|$, by Proposition 3.6(i) we get

$$
\left|a_{i}\right| \leq\|\eta\|_{\infty} \leq 3\|\phi\|_{\infty} \leq \frac{3}{|\Gamma|}, \quad 0 \leq i \leq n .
$$

Therefore in case (II-a) we get

$$
\left\|\psi^{*}\right\|_{\infty} \leq \max _{k-1 \leq i \leq l+1}\left|a_{i}\right| \leq \frac{3}{|\Gamma|} \leq \frac{C_{\gamma}}{|\widetilde{\Delta}|}
$$

(II-b) $k+1 \leq l \leq k+3$. In this case, by strong regularity for pairs we get

$$
\lambda_{n, i}+\lambda_{n, i+1} \sim_{\gamma}|\widetilde{\Delta}|, \quad k-1 \leq i \leq l+1 .
$$

Moreover, from $\|\phi\|_{1} \leq 1$ and Proposition 3.6(i) we deduce that

$$
\sum_{i=k-1}^{l+1}\left|a_{i}\right|\left(\lambda_{n, i}+\lambda_{n, i+1}\right) \leq \sum_{i=0}^{n}\left|a_{i}\right|\left(\lambda_{n, i}+\lambda_{n, i+1}\right) \sim\|\eta\|_{1} \leq 3\|\phi\|_{1} \leq 3 .
$$

These considerations imply that

$$
\left|a_{i}\right| \leq \frac{C_{\gamma}}{|\widetilde{\Delta}|} \quad \text { for } k-1 \leq i \leq l+1
$$

Thus, also in case (II-b) we get

$$
\left\|\psi^{*}\right\|_{\infty} \leq \max _{k-1 \leq i \leq l+1}\left|a_{i}\right| \leq \frac{C_{\gamma}}{|\widetilde{\Delta}|}
$$

Combining (5.12), (5.13) in case (II-a) and (5.14) in case (II-b) and setting

$$
\widetilde{\psi}=\frac{1}{C_{\gamma}} \psi^{*}, \quad b=C_{\gamma},
$$

we find that $\widetilde{\psi}$ is an atom.

It follows from the above considerations (cf. (5.3), (5.9), (5.10), (5.11) and (5.15)) that

$$
\eta=\sum_{i=0}^{k-1} b_{i} \widetilde{\psi}_{i}+b \widetilde{\psi}+\sum_{i=l+1}^{n} b_{i} \widetilde{\psi}_{i}, \quad \sum_{i=0}^{k-1}\left|b_{i}\right|+|b|+\sum_{i=l+1}^{n}\left|b_{i}\right| \leq C_{\gamma}
$$

where all $\widetilde{\psi}, \widetilde{\psi}_{i}, i \leq k-1$ or $i \geq l+1$, are atoms. Thus, formula (5.16) gives an atomic decomposition of $\eta$.

To complete the proof, let $f \in H^{1}[0,1]$. Let $f=\sum_{j=0}^{\infty} d_{j} \phi_{j}$ be an atomic decomposition of $f$ such that $\sum_{j=0}^{\infty}\left|d_{j}\right| \leq 2\|f\|_{H^{1}}$. Without loss of generality, we may assume that $\phi_{0} \equiv 1, d_{0}=\int_{0}^{1} f(t) d t$ and $\phi_{j} \not \equiv 1$ for $j \geq 1$. Let 
$\widetilde{f}=Q_{\pi_{n}} f$ and $\eta_{j}=Q_{\pi_{n}} \phi_{j}$. Since the atomic decomposition of $f$ converges to $f$ in $L^{1}[0,1]$ and the linear operator $Q_{\pi_{n}}$ is bounded on $L^{1}[0,1]$, we have $\widetilde{f}=\sum_{j=0}^{\infty} d_{j} \eta_{j}$, with the series convergent in $L^{1}[0,1]$.

For $j=0, \eta_{0} \equiv 1$ is an atom. For $j \geq 1$, let $\eta_{j}=\sum_{i} b_{i, j} \widetilde{\psi}_{i, j}$ be an atomic decomposition of $\eta_{j}$ having at most $n+1$ nonzero terms and with $\sum_{i}\left|b_{i, j}\right| \leq C_{\gamma}$; for example, it can be the decomposition given by (5.16). Then we have the following atomic decomposition of $\tilde{f}$ :

$$
\tilde{f}=y_{0} \eta_{0}+\sum_{i, j} y_{i, j} \widetilde{\psi}_{i, j}, \quad \text { where } \quad y_{0}=d_{0}, y_{i, j}=d_{j} b_{i, j} \quad \text { for } j \geq 1 .
$$

Observe that

$$
\left|y_{0}\right|+\sum_{i, j}\left|y_{i, j}\right|=\left|d_{0}\right|+\sum_{j}\left|d_{j}\right| \sum_{i}\left|b_{i, j}\right| \leq C_{\gamma} \sum_{j \geq 0}\left|d_{j}\right| \leq 2 C_{\gamma}\|f\|_{H^{1}} .
$$

This implies $\|\widetilde{f}\|_{H^{1}} \leq 2 C_{\gamma}\|f\|_{H^{1}}$, and the proof of Lemma 5.2 is complete.

5.2. The proof of Theorem 2.2. We start with the sufficiency part. Clearly, if $\mathcal{T}$ satisfies the strong regularity condition, then it also satisfies the strong regularity condition for pairs, so by Theorem 2.1, the corresponding Franklin system $\left\{f_{n}, n \geq 0\right\}$ is a basis in $H^{1}[0,1]$. Let $f \in H^{1}[0,1]$, $f=\sum_{n=0}^{\infty} a_{n} f_{n}, \varepsilon=\left(\varepsilon_{n}, n \geq 0\right)$ with $\varepsilon_{n} \in\{-1,1$.$\} . We need to prove the$ convergence in $H^{1}[0,1]$ of the series $f_{\varepsilon} \sim \sum_{n=0}^{\infty} \varepsilon_{n} a_{n} f_{n}$.

It follows from Proposition 4.5 that $\operatorname{Pf} \in L^{1}[0,1]$ and $\|P f\|_{1} \leq$ $C_{\gamma}\|f\|_{H^{1}}$. Since $\operatorname{Pf}=P f_{\varepsilon}$, applying Propositions 4.3 and 4.7 to the sequence of partial sums of the series defining $f_{\varepsilon}$ we find that it is a Cauchy sequence in $H^{1}[0,1]$, Consequently, the series defining $f_{\varepsilon}$ converges in $H^{1}[0,1]$, $f_{\varepsilon} \in H^{1}[0,1]$ and

$$
\left\|f_{\varepsilon}\right\|_{H^{1}} \leq C_{\gamma}\left\|S f_{\varepsilon}\right\|_{1} \leq C_{\gamma}\left\|P f_{\varepsilon}\right\|_{1}=C_{\gamma}\|P f\|_{1} \leq C_{\gamma}\|f\|_{H^{1}} .
$$

This implies that $\left\{f_{n}, n \geq 0\right\}$ is an unconditional basis in $H^{1}[0,1]$.

Now we turn to the necessity part. If $\mathcal{T}$ does not satisfy the strong regularity condition for pairs, then by Theorem 2.1 the corresponding Franklin system is not a basis in $H^{1}[0,1]$. It remains to consider the case when $\mathcal{T}$ satisfies the strong regularity condition for pairs, but fails the strong regularity condition. Then the corresponding Franklin system is a basis in $H^{1}[0,1]$, and we need to show that this basis is not unconditional.

Suppose it is unconditional. Let $f \in H^{1}[0,1], f=\sum_{n=0}^{\infty} a_{n} f_{n}$ and $\varepsilon=\left(\varepsilon_{n}, n \geq 0\right)$ with $\varepsilon_{n} \in\{-1,1$. $\}$. By unconditionality of $\left\{f_{n}, n \geq 0\right\}$ in $H^{1}[0,1]$ we get $f_{\varepsilon}=\sum_{n=0}^{\infty} \varepsilon_{n} a_{n} f_{n} \in H^{1}[0,1]$ (with the series convergent in $\left.H^{1}[0,1]\right)$. Since $\|\cdot\|_{1} \leq\|\cdot\|_{H^{1}}$, this implies that $\sum_{n=0}^{\infty} a_{n} f_{n}$ converges unconditionally in $L^{1}[0,1]$, so by Fact $4.2, P f \in L^{1}[0,1]$. Moreover, by Fact 4.2 
and unconditionality of $\left\{f_{n}, n \geq 0\right\}$ in $H^{1}[0,1]$ we get, for each $f \in H^{1}[0,1]$,

$$
\|P f\|_{1} \leq C \sup _{\varepsilon}\left\|f_{\varepsilon}\right\|_{1} \leq C \sup _{\varepsilon}\left\|f_{\varepsilon}\right\|_{H_{1}} \leq C_{\mathcal{T}}\|f\|_{H^{1}}
$$

with the constant $C_{\mathcal{T}}$ independent of $f$. But it follows from Proposition 4.6 that inequality (5.17) does not hold, even for atoms. This contradiction completes the proof.

Corollary 5.3. Let $\mathcal{T}$ be an admissible sequence of knots in $[0,1]$ satisfying the strong regularity condition with parameter $\gamma$. Let $a=\left(a_{n}, n \geq 0\right)$ be a sequence of coefficients. Then conditions (A)-(D) from Section 4 are equivalent. Moreover, they are equivalent to the following condition (E):

(E) The series $\sum_{n=0}^{\infty} a_{n} f_{n}$ converges unconditionally in $H^{1}[0,1]$.

In addition, for $f \in H^{1}[0,1], f=\sum_{n=0}^{\infty} a_{n} f_{n}$, we have

$$
\|f\|_{H^{1}} \sim_{\gamma}\|P f\|_{1} \sim_{\gamma}\|S f\|_{1} \sim_{\gamma} \sup _{\varepsilon=\left(\varepsilon_{n}, n \geq 0\right), \varepsilon_{n} \in\{-1,1\}}\left\|\sum_{n=0}^{\infty} \varepsilon_{n} a_{n} f_{n}\right\|_{1},
$$

with the implied constants depending only on $\gamma$.

Finally, let us remark that when an admissible sequence of knots satisfies the strong regularity condition, then the corresponding Franklin system is a greedy basis in $H^{1}[0,1]$. For the definition of greedy basis see [12], where this notion has been introduced, or e.g. to [10], where we have checked that each general Franklin system (properly normalized) is a greedy basis in $L^{p}[0,1]$, $1<p<\infty$.

COROLlary 5.4. Let $\mathcal{T}$ be an admissible sequence of knots in $[0,1]$ satisfying the strong regularity condition with parameter $\gamma$. Then $\left\{f_{n} /\left\|f_{n}\right\|_{H^{1}}\right.$, $n \geq 0\}$, the corresponding Franklin system, normalized in $H^{1}[0,1]$, is a greedy basis in $H^{1}[0,1]$.

Proof. We just give a sketch of the proof, because it follows the lines of the proof of Corollary 2.2 in [10].

It has been proved in [12] that a basis in a Banach space is greedy if and only if it is unconditional and democratic (see [12] or [10] for the definition). Theorem 2.2 guarantees that for strongly regular sequences of knots, $\left\{f_{n} /\left\|f_{n}\right\|_{H^{1}}, n \geq 0\right\}$ is an unconditional basis in $H^{1}[0,1]$. To prove that this system is democratic, we check that for each $m$ and $n_{1}<\cdots<n_{m}$,

$$
\left\|\frac{f_{n_{1}}}{\left\|f_{n_{1}}\right\|_{H^{1}}}+\cdots+\frac{f_{n_{m}}}{\left\|f_{n_{m}}\right\|_{H^{1}}}\right\|_{H^{1}} \sim_{\gamma} m .
$$

But it follows from Corollary 5.3 that $\left\|f_{n}\right\|_{H^{1}} \sim\left\|f_{n}\right\|_{1}$. Therefore, the equivalence (5.18) is checked in the same way as the democracy of $\left\{f_{n} /\left\|f_{n}\right\|_{p}, n \geq 0\right\}$ in $L^{p}[0,1]$ (cf. the proof of Corollary 2.2 in [10]), with the use of $\|f\|_{H^{1}} \sim_{\gamma}$ $\|P f\|_{1}$ and Proposition 4.5 of [10]. 


\section{References}

[1] S. V. Bochkarev, Some inequalities for the Franklin series, Anal. Math. 1 (1975), 249-257.

[2] L. Carleson, An explicit unconditional basis in $H^{1}$, Bull. Sci. Math. (2) 104 (1980), 405-416.

[3] S.-Y. A. Chang and Z. Ciesielski, Spline characterizations of $H^{1}$, Studia Math. 75 (1983), 183-192.

[4] Z. Ciesielski, Properties of the orthonormal Franklin system, ibid. 23 (1963), 141157.

[5] Z. Ciesielski and A. Kamont, Projections onto piecewise linear functions, Funct. Approx. Comment. Math. 25 (1997), 129-143.

[6] R. R. Coifman, A real variable characterization of $H^{p}$, Studia Math. 51 (1974), 269-274.

[7] Ph. Franklin, A set of continuous orthogonal functions, Math. Ann. 100 (1928), 522-528.

[8] G. G. Gevorkyan, Some theorems on unconditional convergence and the majorant of Franklin series and their application to Re $H_{p}$, Trudy Mat. Inst. Steklova 190 (1989), 49-74 (in Russian); English transl.: Proc. Steklov Inst. Math. 1992, 49-76.

[9] G. G. Gevorkyan and A. Kamont, On general Franklin systems, Dissertationes Math. 374 (1998).

[10] - - - Unconditionality of general Franklin system in $L^{p}[0,1], 1<p<\infty$, Studia Math. 164 (2004), 161-204.

[11] G. G. Gevorkyan and A. A. Sahakian, Unconditional basis property of general Franklin systems, Izv. Nats. Akad. Nauk Armenii Mat. 35 (2000), no. 4, 7-25 (in Russian); English transl.: J. Contemp. Math. Anal. 35 (2000), no. 4, 2-22.

[12] S. V. Konyagin and V. N. Temlyakov, A remark on greedy approximation in Banach spaces, East J. Approx. 5 (1999), 1-15.

[13] B. Maurey, Isomorphismes entre espaces $H_{1}$, Acta Math. 145 (1980), 79-120.

[14] Y. Meyer, Wavelets and Operators, Cambridge Univ. Press, Cambridge, 1992.

[15] P. Sjölin, Convergence almost everywhere of spline expansions in Hardy spaces, in: Topics in Modern Harmonic Analysis (Turin/Milan, 1982), Ist. Naz. Alta Mat. Francesco Severi, Roma, 1983, 645-651.

[16] P. Sjölin and J. O. Strömberg, Basis properties of Hardy spaces, Ark. Mat. 21 (1983), 111-125.

[17] P. Wojtaszczyk, The Franklin system is an unconditional basis in $H^{1}$, ibid. 20 (1982), 293-300.

[18] —, A Mathematical Introduction to Wavelets, Cambridge Univ. Press, Cambridge, 1997.

Department of Mathematics

Yerevan State University

Alex Manoukian St. 1

375049 Yerevan, Armenia

E-mail: ggg@arminco.com
Institute of Mathematics Polish Academy of Sciences

Abrahama 18

81-825 Sopot, Poland

E-mail: A.Kamont@impan.gda.pl 